\title{
Albino Canfora
}

Extension of an integral inequality of C. Miranda and applications to the elliptic equations with discontinuous coefficients

Czechoslovak Mathematical Journal, Vol. 39 (1989), No. 3, 385-422

Persistent URL: http://dml.cz/dmlcz/102312

\section{Terms of use:}

(C) Institute of Mathematics AS CR, 1989

Institute of Mathematics of the Czech Academy of Sciences provides access to digitized documents strictly for personal use. Each copy of any part of this document must contain these Terms of use.

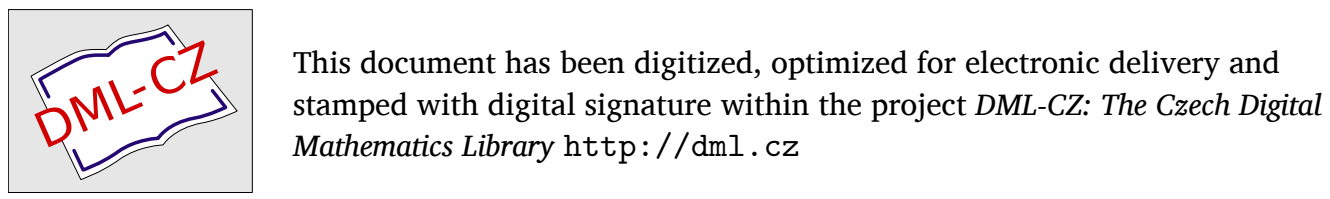




\title{
EXTENSION OF AN INTEGRAL INEQUALITY OF C. MIRANDA AND APPLICATIONS TO THE ELLIPTIC EQUATIONS WITH DISCONTINUOUS COEFFICIENTS
}

\author{
Albino Canfora, Naples
}

(Received September 23, 1986)

\section{INTRODUCTION}

In a classical paper of 1963 [7], C. Miranda established some generalization of the integral inequalities of Sobolev, Gagliardo and Nirenberg.

The starting point was the inequality described below concerning the functions of one variable defined in $I=] 0,1$ ( (see Lemma 1.I and 1.II of [7]).

Let $\alpha, p, p_{0}, \eta$ be real numbers such that:

$$
\begin{gathered}
\alpha \geqq 0 ; \quad p \geqq 1 ; \quad p_{0} \geqq 0 ; \\
1 \leqq q_{1} \equiv \frac{p p_{0}(\alpha+2)}{p+p_{0}} \leqq \eta(\alpha+2) ;
\end{gathered}
$$

then there exists a constant $K$ depending only on $\eta$ such that:

$$
\left|u^{\prime}\right|_{I, q_{1}} \leqq K\left[\left.\left.|| u^{\prime}\right|^{\alpha} u^{\prime \prime}\right|_{I, p} ^{1 /(\alpha+2)}|u|_{I, p_{0}}^{1 /(\alpha+2)}+|u|_{I, p_{0}}\right]\left(|u|_{J, s}=\|u\|_{L^{s}(J)}\right)
$$

for every function $u \in C^{2}(I)$ for which the right-hand side is finite.

In (2) the second addendum, in the right-hand side can be omitted if $u^{\prime}$ is zero in at least one point of $I=] 0,1[$.

The inequality (2), if $\alpha=0$, becomes the inequality established, at the some time, by E. Gagliardo and L. Nirenberg in [4] and [11].

The right-hand side is always finite if we consider only functions $u$ continuous together with their first and second derivatives in the whole $\bar{I}=[0,1]$.

This is the starting case for the proof of the Lemma 2.II of [7]. Thus (2) gives

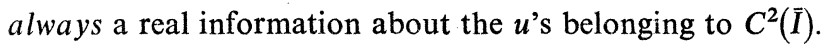

The problem we deal with, in this paper is the following: how can (2) be extended if $\alpha<0$ ? 
The "natural" extension of (2) to the case $\alpha<0$ seems the following:

$$
\begin{gathered}
\left(\int_{E}\left|u^{\prime}\right|^{q_{1}} \mathrm{~d} x\right)^{1 / q_{1}} \leqq K\left[\left(\int_{E^{0} u^{\prime}}\left|u^{\prime}\right|^{\alpha p}\left|u^{\prime \prime}\right|^{p} \mathrm{~d} x\right)^{1 / p(\alpha+2)}\left(\int_{E}|u|^{p_{0}} \mathrm{~d} x\right)^{1 / p_{0}(\alpha+2)}+\right. \\
\left.+\left(\int_{E}|u|^{p_{0}} \mathrm{~d} x\right)^{1 / p_{0}}\right]
\end{gathered}
$$

where: $E=\bar{I}=[0,1]$ and $E_{u^{\prime}}^{\tau}=\left\{x \in E:\left|u^{\prime}(x)\right|>\tau\right\}(\tau \geqq 0)$.

Nevertheless, when $\alpha<0$, however small $|\alpha|$ is, it is always possible to find $u \in C^{2}(\dot{E})$ for which the right-hand side of (2)' is not finite. For example (see Remark 1.III), for $\alpha=-3 / 2(k+2)(k$ integer $\geqq 1), p=2$ and any $p_{0}$, the function:

$$
\begin{gathered}
u(x)=\psi(x) \int_{0}^{x} t^{k+2} \operatorname{sen}^{2}\left(t^{-k}\right) \mathrm{d} t \\
{\left[u(0)=0 ; \text { with: } \psi \in C_{0}^{\infty}(]-1,1[), \psi \equiv 1 \text { for }|x| \leqq \frac{1}{2}\right]}
\end{gathered}
$$

belongs to $C^{2}(E)$, but makes infinite the right-hand side of $(2)^{\prime}$. We observe that the function $u(x)$ of (3) vanishes in 0 and in 1 ; so that we can say that $(2)^{\prime}$ gives an aleatory information even if we let $u$ vary in the class

$$
\mathscr{U}=\left\{u \in C^{2}(E): u(0)=u(1)=0\right\} .
$$

In place of (2)' it would be desirable an inequality where, at least for $u \in \mathscr{U}$, the righthand side is always finite even when $\alpha<0$.

To do this it is reasonable to try to estimate the left-hand side of $(2)^{\prime}$ substituting the integral:

$$
\int_{E^{0} u^{\prime}}\left|u^{\prime}\right|^{\alpha p}\left|u^{\prime \prime}\right|^{p} \mathrm{~d} x \quad\left(E_{u^{\prime}}^{0}=\left\{x \in E:\left|u^{\prime}(x)\right|>0\right\}\right)
$$

which appear in the right-hand side, with the integral, certainly finite:

$$
\int_{E^{\tau} u^{\prime}}\left|u^{\prime}\right|^{\alpha p}\left|u^{\prime \prime}\right|^{p} \mathrm{~d} x \quad\left(E_{u^{\prime}}^{\tau}=\left\{x \in E:\left|u^{\prime}(x)\right|>\tau\right\}\right)
$$

with a suitable $\tau>0$ [if $u \neq 0$ ]. But does such a $\tau$ exist?

Actually we succed in proving that (see Theorem 1.I):

$$
\begin{gathered}
\left(\int_{E}\left|u^{\prime}\right|^{q_{1}} \mathrm{~d} x\right)^{1 / q_{1}} \leqq \\
\leqq \bar{K}_{1}\left[\left(\int_{E_{u^{\prime}}^{\beta_{u}}}\left|u^{\prime}\right| p^{p \alpha}\left|u^{\prime \prime}\right|^{p} \mathrm{~d} x\right)^{1 / p(\alpha+2)}\left(\int_{E}|u|^{p_{0}} \mathrm{~d} x\right)^{1 / p_{0}(\alpha+2)}+\left(\int_{E}|u|^{p_{0}} \mathrm{~d} x\right)^{1 / p_{0}}\right], \\
\forall u \in U
\end{gathered}
$$

where: $E=[a, b], \mathscr{U}=\left\{u \in C^{2}(E): u(a)=u(b)=0\right\}$,

$$
\begin{gathered}
\tau \equiv \beta_{u}=|u|_{E, p_{0}}(\text { meas } E)^{-1 / q_{1}}, \\
E_{u^{\prime}}^{\beta_{u}}=\left\{x \in E:\left|u^{\prime}(x)\right|>\beta_{u}\right\}, \quad\left[E_{u^{\prime}}^{\beta_{u}}=\emptyset, \text { if } u \equiv 0\right],
\end{gathered}
$$

and, in place of (1), the following conditions hold:

$$
\begin{gathered}
p, p_{0}>1 ; \quad 1>\frac{1}{p}+\frac{1}{p_{0}}>\eta>0 ; \quad \gamma \geqq 0 ; \\
\gamma>\alpha>\left(\frac{1}{p}+\frac{1}{p_{0}}\right)(1+\eta)-2 .
\end{gathered}
$$

As for (2), also in (4): $q_{1}=p p_{0}(\alpha+2) /\left(p+p_{0}\right)(>1+\eta)$. 
The difference is that now $\alpha$ can be negative [it can approach -2 ]. The constant $\bar{K}_{1}$ which appear in (4) is given by:

$$
\bar{K}_{1}=2^{1+(1 / \eta)+\left(1 / \eta^{2}\right)}\left(\frac{\gamma+2}{\eta}\right)^{1 / \eta^{2}} .
$$

If $[a, b]=[0,1]$ the function $u(x)$ of (3) belongs to the class $\mathscr{U}$, so that $(4)-$ unlike $(2)^{\prime}-$ gives a real information about $u(x)$.

Besides (4), we prove the inequalities (see (1.6) and (1.9)):

$$
\begin{gathered}
\left|u^{\prime}\right|_{E, q_{1}} \leqq \bar{K}\left[\left.\left.|| u^{\prime}\right|^{\alpha} u^{\prime \prime}\right|_{E^{1} u^{\prime}, p} ^{1 /(\alpha+2)}|u|_{E^{1} u^{\prime}, p_{0}}^{1 /(\alpha+2)}+\left|u^{\prime}\right|_{E, 1}^{1 / q_{1}}\right], \quad \forall u \in \mathscr{U}, \\
\left|u^{\prime}\right|_{E, q_{1}} \leqq \bar{K}_{1}\left[\left.\left.|| u^{\prime}\right|^{\alpha} u^{\prime \prime}\right|_{E^{1} u^{\prime}, p} ^{1 /(\alpha+2)}|u|_{E^{1} u^{\prime}, p_{0}}^{1 /(\alpha+2)}+(\operatorname{meas} E)^{1 / q_{1}}\right], \quad \forall u \in \mathscr{U},
\end{gathered}
$$

where: $\bar{K}=2^{1 / \eta}((\gamma+2) / \eta)^{1 / \eta}$.

All these inequalities are proved by elementary procedures like those used by E. Gagliardo in [4].

The hold also for subset of $C^{2}(E)$ different from $\mathscr{U}$; for example (see Lemma 1.I), (6) holds also in

$$
\left\{u \in C^{2}(E):\left|u^{\prime}(a)\right|,\left|u^{\prime}(b)\right| \leqq 1\right\} .
$$

It is still open the problem to see what can be obtained, in general, for $u \in C^{2}(E)$ : to this regard see the Remark 1.II. It is also open the problem to establish the inequality analogous to (4) for the functions belonging to $C^{k}(E)$, for any $k$.

The same problems hold for the functions of several variables defined in an open set $\Omega \subset \mathbb{R}^{n}$, for which in the present paper only some inequalities are proved (section 2 ) in view of the applications.

For functions of several variables there are also questions concerning the necessary regularity for $\partial \Omega$.

In section 2 we assume $\Omega$ convex (bounded and open), but C. Miranda's inequalities [7] are proved assuming only that $\Omega$ verifies a cone property.

One could also investigate about "best constant" in all these inequalities. It would be also desirable to improve some of the inequalities which are (see for instance Theorem 2.II) definitely farraginous, even if suitable for the main purpose of this paper. The main purpose of this paper is to study an elliptic equation whose coefficients are not continuous.

For this kind of equation (see section 4) we prove an existence theorem (for the Dirichlet problem in a cube) applying the inequalities of section 2 . For those of section 1 (concerning functions of one variable) an application is given in the same section 1 (see Remark I.IV) where an estimate for the mean variation of order $q$ of a function $f \in C^{1}(E)=C^{1}([a, b])$ is given.

The inequality we obtain is the following:

$$
\begin{gathered}
\mu_{q, E}(f) \leqq \bar{K}_{1}+ \\
+\bar{K}_{1}\left(\operatorname{meas} E^{1}\right)^{1 / q}\left\{2(\operatorname{meas} E)^{1-((\alpha+2) / q)} M_{p_{0}, E}(f) M_{p, E^{1}}\left(\frac{\left|f^{\prime}\right|}{\left|f-m_{E}(f)\right|^{|\alpha|}}\right)\right\}^{1 /(\alpha+2)}
\end{gathered}
$$


where: $p, p_{0}, \eta, \alpha<0 \equiv \gamma$ satisfy (5) and:

$$
\begin{gathered}
q \equiv q_{1}=\frac{p p_{0}(\alpha+2)}{p+p_{0}}, \quad E^{1}=\left\{x \in E:\left|f(x)-m_{E}(f)\right|>1\right\}, \\
m_{E}(f)=(\text { meas } E)^{-1} \int_{E} f(x) \mathrm{d} x, \\
\mu_{q, E}(f)=\left[(\text { meas } E)^{-1} \int_{E}\left|f(x)-m_{E}(f)\right|^{q} \mathrm{~d} x\right]^{1 / q}, \\
M_{r, B}(g)=\left[(\text { meas } B)^{-1} \int_{B}|g(x)|^{r} \mathrm{~d} x\right]^{1 / r} .
\end{gathered}
$$

Inequality (8) comes from (7).

Let us say something about the equation we mentioned above. In the basic paper [8] of 1963, C. Miranda established (among other things) some existence and uniqueness theorems for the equation:

$$
\sum_{i, k=1}^{n} a_{i k} D_{x_{i}} D_{x_{k}} u+\sum_{i=1}^{n} b_{i} D_{x_{i}} u+c u=f, \quad \text { in }\left.\quad \Omega \quad u\right|_{\partial \Omega}=0
$$

where $\Omega$ is a bounded open set of $R^{n}$, belonging to $C^{3}$ and:

$$
\begin{aligned}
m_{0}|\xi|^{2} & \leqq \sum_{i, k} a_{i k} \xi_{i} \xi_{k} \leqq m_{1}|\xi|^{2}, \quad \forall \xi \in \mathbb{R}^{n} \\
m_{0} & >0, \quad|\xi|^{2}=\xi_{1}^{2}+\ldots+\xi_{n}^{2}
\end{aligned}
$$

Precisely the results are the folowing:

(i) (see Theorem 1.V of [8]). If, besides $(9)^{\prime}$, we have:

$(9)^{\prime \prime} \quad c(x) \leqq c_{0}<0$;

A) $a_{i k}(x) \in W^{1, n}(\Omega), \quad$ B) $\quad b_{i}(x) \in L^{n}(\Omega), \quad c(x) \in L^{n / 2}(\Omega)$,

then, for each $f \in L^{2}(\Omega)$, equation (9) has a unique solution $u \in W^{2,2}(\Omega)$.

(ii) (se Theorem 5.V of [8]). If, besides (9)', we have:

$\left.\left.(9)^{\prime \prime \prime} \quad c(x) \leqq c_{0}<0 ; \quad \mathrm{A}_{1}\right) \quad a_{i k}(x) \in W^{1, \lambda}(\Omega), \quad \mathrm{B}_{1}\right) \quad b_{i}(x) \in L^{\lambda}(\Omega), c(x) \in L^{\lambda / 2}(\Omega)$,

with $4 \leqq \lambda<n$, then, for each $f \in L^{\infty}(\Omega)$, equation (9) has, at least one solution $u \in W^{2,2}(\Omega) \cap L^{\infty}(\Omega)$. When $\lambda<n$, the first hypothesis of $(9)^{\prime \prime \prime}$ does not assure the uniqueness any more (see the counterexample of Alexandrov, mentioned also in [9]).

The crucial point of the proof of Theorems $1 . \mathrm{V}$ and $5 . \mathrm{V}$ of [8] is the estimate of the integral (2.14).

For the Theorem 1.V this estimate follows essentially from Sobolev's Theorem which requires condition A) $a_{i k} \in W^{1, n}$.

For the Theorem 5.V, instead, the estimate of the integral (2.14) is obtained using (5.5) [8] (that is (2)) which allows the weaker hypothesis $\mathrm{A}_{1}$ ) $a_{i k} \in W^{1, \lambda}$.

Then one can ask whether the use of an inequality like (2), but with $\alpha<0$, could allow even less restrictive hypotheses on the coefficients reducing the summability of their derivatives to an exponent smaller than 4 .

That was the first idea which brought us to extend the inequality (2) to the case $\alpha<0$. 
Once such an extension was obtained, the plan we had in mind effectively worked.

Actually we consider, in sections 3 and 4 an an elliptic equation with coefficients belonging to $W^{1, \lambda}(\lambda<4)$ and prove for it an existence theorem.

The equation we deal with has the simplest form in order to get rid of additional difficulties.

Similarly, to avoid complications in writing (2.11) of [8] when $\alpha<0$, we set the problem in the cube $\Omega=] 0,1\left[{ }^{n}\right.$. But it is clear that these aspects of the problem should also be examined.

The theorem we prove (Theorem 4.II) is the following: Let $\Omega$ be $] 0,1\left[{ }^{n}\right.$ and $a_{1}(x), \ldots$ $\ldots, a_{n}(x)$ functions such that:

$$
a_{i} \in W^{1,4+2 \alpha}(\Omega) \text { with } \quad-\frac{1}{6} \leqq \alpha<0 ; \quad \inf _{\Omega} a_{i}(x)>0 ;
$$

then, for each $f \in L^{\infty}(\Omega)$, there exists at least one $u \in W^{2,2+\alpha}(\Omega) \cap L^{\infty}(\Omega)$ such that:

$$
\begin{gathered}
\mathscr{L} u \equiv-\sum_{i=1}^{n} a_{i}(x) D_{x_{i}}^{2} u=f(x) \text { in } \Omega, \\
\left.u\right|_{\partial \Omega}=0 .
\end{gathered}
$$

The general scheme of the proof is that of $C$. Miranda, but the condition $\alpha<0$ creats additional difficulties so that it has been necessary to show the proof in detail.

It should be remarked that assumptions (10) do not require the coefficients to be bounded.

A concrete example of an equation to which Theorem 4.II applies (see section 6) is, in $R^{4}$, the following:

$$
-\sum_{i=1}^{4}\left(1+\left|x-x^{i}\right|^{-1 /(22+i)}\left[1+\operatorname{sen}\left(\left|x-x^{i}\right|^{-1 /(22+i)}\right)\right]\right) D_{x_{i}}^{2} u(x)=f(x)
$$

where $x^{i}=\left(x_{1}^{i}, \ldots, x_{4}^{i}\right)$ is a fixed point of $\left.\Omega=\right] 0,1\left[{ }^{4}\right.$. Another examples is furnished, in $R^{5}$, by the equation:

$$
-\sum_{i=1}^{5}\left(1+\delta_{i}(x)^{-1 /(22+i)}\left[1+\operatorname{sen}\left(\delta_{i}(x)^{-1 /(22+i)}\right)\right]\right) D_{x_{i}}^{2} u(x)=f(x)
$$

where: $\delta_{i}(x)=\left[\sum_{j \neq i} x_{j}^{2}\right]^{1 / 2}$ is the distance of $x$ from the $x_{i}$ axis.

Acknowledgement. I am very grateful to my dear colleague Filomena Pacella for some useful talks about the subject of this paper.

\section{SOME INEQUALITIES FOR FUNCTIONS OF ONE VARIABLE}

From now on we will consider real numbers $\eta, \gamma ; p, p_{0}, \alpha$, satisfying the following conditions:

$$
\begin{gathered}
p>1, \quad p_{0}>1, \quad \gamma \geqq 0, \\
1>\frac{1}{p}+\frac{1}{p_{0}}>\eta>0, \quad \gamma>\alpha>\left(\frac{1}{p}+\frac{1}{p_{0}}\right)(1+\eta)-2 .
\end{gathered}
$$


From (1.1) we have immediately:

$$
\begin{gathered}
p p_{0}>p+p_{0}>2,0<\eta<1, \\
\frac{1}{\eta}>\frac{p p_{0}}{p+p_{0}} \equiv \frac{1}{1-\left(1-\frac{p+p_{0}}{p p_{0}}\right)}, \\
\frac{\gamma+2}{\eta}>\frac{p p_{0}(\alpha+2)}{p+p_{0}} \equiv q>1+\eta, \\
\left(\frac{\gamma+2}{\eta}\right)^{1 / \eta}>(1+\eta)^{1 / \eta}>2 .
\end{gathered}
$$

It is useful to observe that, if $\alpha, \eta, p$ satisfy:

$$
\begin{gathered}
p>1, \quad 1>\frac{1}{p}>\eta>0, \\
0>\alpha>\frac{1+\eta}{p}-2 ;
\end{gathered}
$$

then, for

$$
p_{0} \geqq \bar{p}>\max \left\{\frac{p}{p-1}, \frac{(1+\eta) p}{p(\alpha+2)-1-\eta}\right\}
$$

the numbers $\eta, \gamma=0 ; p, p_{0}, \alpha$ satisfy (1.1).

Among the numbers for which $(1.1)_{\infty}$ is true there are, for instance, $p=2$; $\left.\eta=\frac{1}{4} ; \alpha \in\right]_{\frac{5}{8}}-2,0[$.

The following inequalities will be often used:

$$
\begin{gathered}
\left\{0 \leqq \xi<\sum_{i=1}^{n} a_{i} \xi^{x_{i}} \text {, with: } a_{i}>0 \leqq \alpha_{i}<1\right\} \Rightarrow \\
\Rightarrow\left\{\xi<\sum_{i=1}^{n}\left(q_{i} a_{i}\right)^{1 /\left(1-\alpha_{i}\right)}, \quad \forall\left(q_{1}, \ldots, q_{n}\right) \in \mathbb{R}^{n} \text { with } q_{i}>0 \text { and } \sum_{i=1}^{n} \frac{1}{q_{i}}=1\right\} ; \\
\{a, b, s>0\} \Rightarrow\left\{(a+b)^{s} \leqq 2^{s}\left(a^{s}+b^{s}\right)\right\} .
\end{gathered}
$$

If $A$ is an open set in $R^{n}$ we will denote by $C^{k}(\bar{A})$ the set of functions $u$ which can be extended, together with their derivatives, up to the order $k$, continuously to the closure $\bar{A}$ of $A$.

If $\varphi(x)$ is measurable in $A$ and $\tau \geqq 0$ is a real number, we set: $A_{\varphi}^{\tau}=\{x \in A$ : $|\varphi(x)|>\tau\}$. Obviously $A_{\varphi}^{\tau}=\emptyset$ if $|\varphi(x)| \leqq \tau, \forall x \in A$. For sake of simplicity we will write $A^{\tau}$ and $A_{i, \varphi}^{\tau}$ instead of $A_{\varphi}^{\tau}$ and $A_{D_{x_{i} \varphi}}^{\tau}$ respectively, if there is not misundersstanding.

Finally we will use the notation:

$$
\begin{gathered}
|u|_{A, s}=\|u\|_{L^{*}(A)} \equiv\left(\int_{A}|u(x)|^{s} \mathrm{~d} x\right)^{1 / s}, \\
\left|D^{k} u\right|_{A, s}=\sum_{|\alpha|=k}\left|D^{\alpha} u\right|_{A, s} .
\end{gathered}
$$


Lemma 1.I. Let $E$ be the interval $[a, b] \subset R$ and $\eta, \gamma ; p, p_{0}, \alpha$ real numbers satisfying (1.1).

Then, for every $u \in C^{2}(E)$, with $\left|u^{\prime}(a)\right|,\left|u^{\prime}(b)\right| \leqq 1$, we have:

$$
\left|u^{\prime}\right|_{E, q} \leqq \bar{K}\left[\|\left.\left. u^{\prime}\right|^{\alpha} u^{\prime \prime}\right|_{E^{1} u^{\prime}, p} ^{1 /(\alpha+2)}|u|_{E^{1} u^{\prime}, p_{0}}^{1 /(+2)}+\left|u^{\prime}\right|_{E, 1}^{1 / q}\right],
$$

where:

$$
q=\frac{p p_{0}(\alpha+2)}{p+p_{0}}, \quad \bar{K} \equiv \bar{K}(\gamma, \eta)=2^{1 / \eta}\left(\frac{\gamma+2}{\eta}\right)^{1 / \eta} .
$$

Proof. Since: $\left|u^{\prime}(b)\right|,\left|u^{\prime}(a)\right| \leqq 1$, the set $E_{u^{\prime}}^{1} \equiv\left\{x \in E:\left|u^{\prime}(x)\right|>1\right\}$, eventually empty, is open in $R$ (because of the continuity of $u^{\prime}$ ) and can be written as the disjoint union

$$
\left.E_{u^{\prime}}^{1}=\bigcup_{i=1}^{\infty}\right] a_{i}, b_{i}[\subset] a, b[\text {. }
$$

In the generic interval $] a_{i}, b_{i}\left[\right.$ we have either $u^{\prime}(x)>1 \forall x$ or $u^{\prime}(x)<-1 \forall x$.

If, for example, $\left.u^{\prime}(x)<-1 \forall x \in\right] a_{i}, b_{i}\left[\right.$ then $u^{\prime}\left(a_{i}\right)=u^{\prime}\left(b_{i}\right)=-1$ and, arguing as in Gagliardo's paper [4]:

$$
\begin{aligned}
J_{i} & \equiv \int_{a_{i}}^{b_{i}}\left|u^{\prime}\right|^{q} \mathrm{~d} x=\int_{a_{i}}^{b_{i}}\left|u^{\prime}\right|^{q-1}\left(-u^{\prime}\right) \mathrm{d} x= \\
& =-\left(u\left(b_{i}\right)-u\left(a_{i}\right)\right)+\int_{a_{i}}^{b_{i}} u D\left(\left|u^{\prime}\right|^{q-1}\right) \mathrm{d} x= \\
& =-\int_{a_{i}}^{b_{i}} u^{\prime} \mathrm{d} x+(q-1) \int_{a_{i}}^{b_{i}}\left|u^{\prime}\right|^{q-3} u^{\prime} u^{\prime \prime} u \mathrm{~d} x= \\
& =\int_{a_{i}}^{b_{i}}\left|u^{\prime}\right| \mathrm{d} x+(q-1) \int_{a_{i}}^{b_{i}}\left|u^{\prime}\right|^{\alpha} u^{\prime \prime}\left|u^{\prime}\right|^{q-3-\alpha} u^{\prime} u \mathrm{~d} x,
\end{aligned}
$$

since the formula: $D|f|^{\beta}=\beta|f|^{\beta-2} f f^{\prime}$ is true $\forall \beta \in R$ for the points $x$ where $f(x) \neq 0$ and here $f(x)=u^{\prime}(x) \neq 0 \forall x \in\left[a_{i}, b_{i}\right]$.

So, in the case $\left.u^{\prime}(x)<-1 \forall x \in\right] a_{i}, b_{i}[$ we have:

$$
J_{i} \equiv \int_{a_{i}}^{b_{i}}\left|u^{\prime}\right|^{q} \mathrm{~d} x \leqq \int_{a_{i}}^{b_{i}}\left|u^{\prime}\right| \mathrm{d} x+\left.\left.(q-1) \int_{a_{i}}^{b_{i}}|| u^{\prime}\right|^{\alpha} u^{\prime \prime}|\cdot| u^{\prime}\right|^{\sigma} \cdot|u| \mathrm{d} x,
$$

where:

$$
\begin{gathered}
\sigma=q-2-\alpha \equiv \frac{\left(p p_{0}-p-p_{0}\right)(\alpha+2)}{p+p_{0}}>0 \\
\left(\Rightarrow \sigma \frac{p p_{0}}{p p_{0}-p-p_{0}}=q\right) .
\end{gathered}
$$

Using the same procedure in the intervals where $u^{\prime}(x)>1$, we find that (1.4) holds for any interval $] a_{i}, b_{i}$. Taking the sum over $i$ and applying Holder's inequality to the integrals and the sums we get:

$$
\int_{E_{u^{\prime}}{ }^{\prime}}\left|u^{\prime}\right|^{q} \mathrm{~d} x=\sum_{i=1}^{\infty} J_{i} \leqq
$$

$$
\leqq \sum_{i} \int_{a_{i}}^{b_{i}}\left|u^{\prime}\right| \mathrm{d} x+\left.\left.(q-1) \sum_{i} \int_{a_{i}}^{b_{i}}|| u^{\prime}\right|^{\alpha} u^{\prime \prime}|| u^{\prime}\right|^{\sigma}|u| \mathrm{d} x \leqq \int_{E^{1} u^{\prime}}\left|u^{\prime}\right| \mathrm{d} x+
$$

$+(q-1) \sum_{i}\left(\left.\left.\int_{a_{i}}^{b_{i}}|| u^{\prime}\right|^{\alpha} u^{\prime \prime}\right|^{p} \mathrm{~d} x\right)^{1 / p}\left(\int_{a_{i}}^{b_{i}}|u|^{p_{0}} \mathrm{~d} x\right)^{1 / p_{0}}\left(\int_{a_{i}}^{b_{i}}\left|u^{\prime}\right|^{\sigma} \frac{p p_{0}}{p p_{0}-p-p_{0}} \mathrm{~d} x\right)^{\frac{p p_{0}-p-p_{0}}{p p_{0}}} \leqq \int_{E^{1}{ }_{u^{\prime}}}\left|u^{\prime}\right| \mathrm{d} x+$ 


$$
\begin{aligned}
& +(q-1)\left[\left.\left.\sum_{i} \int_{a_{i}}^{b_{i}}|| u^{\prime}\right|^{\alpha} u^{\prime \prime}\right|^{p} \mathrm{~d} x\right]^{1 / p}\left[\sum_{i} \int_{a_{i}}^{b_{i}}|u|^{p_{0}} \mathrm{~d} x\right]^{1 / p_{0}}\left[\sum_{i} \int_{a_{i}}^{b_{i}}\left|u^{\prime}\right| q \mathrm{~d} x\right]^{\frac{p p_{0}-p-p_{0}}{p p_{0}}} \equiv \\
& \equiv \int_{E^{1} u^{\prime}}\left|u^{\prime}\right| \mathrm{d} x+(q-1)\left(\left.\int_{E^{1} u^{\prime}}|| u^{\prime}\right|^{\alpha} u^{\prime \prime} \mid p \mathrm{~d} x\right)^{1 / p}\left(\int_{E^{1} u^{\prime}}|u|^{p_{0}} \mathrm{~d} x\right)^{1 / p_{0}}\left(\int_{E^{1} u^{\prime}}\left|u^{\prime}\right|^{q} \mathrm{~d} x\right)^{1-\frac{p+p_{0}}{p_{0}}} .
\end{aligned}
$$

Now if we use the first inequality in (1.2)

$$
\text { [with: } \left.q_{1}=q_{2}=2, \quad \xi=\int_{E^{1} u^{\prime}}\left|u^{\prime}\right|^{q} \mathrm{~d} x, \quad \alpha_{1}=0, \quad \alpha_{2}=1-\frac{p+p_{0}}{p p_{0}}<1\right]
$$

and $(1.1)^{\prime}$ we obtain:

$$
\begin{gathered}
\int_{E^{1} u^{\prime}}\left|u^{\prime}\right|^{q} \mathrm{~d} x \leqq 2 \int_{E^{1} u^{\prime}}\left|u^{\prime}\right| \mathrm{d} x+ \\
+\left[2(q-1)\left(\left.\left.\int_{E^{1} u^{\prime}}|| u^{\prime}\right|^{\alpha} u^{\prime \prime}\right|^{p} \mathrm{~d} x\right)^{1 / p}\left(\int_{E^{1} u^{\prime}}|u|^{p_{0}} \mathrm{~d} x\right)^{1 / p_{0}}\right]^{1 /\left(1-\alpha_{2}\right)} \leqq \\
\leqq 2 \int_{E^{1} u^{\prime}}\left|u^{\prime}\right| \mathrm{d} x+2^{1 / \eta}\left(\frac{\gamma+2}{\eta}\right)^{1 / \eta}\left(\left.\int_{E^{1} u^{\prime}}|| u^{\prime}\right|^{\alpha} u^{\prime \prime} \mid p d x\right)^{p_{0} /\left(p+p_{0}\right)}\left(\int_{E^{1} u^{\prime}}|u|^{p_{0}} \mathrm{~d} x\right)^{p /\left(p+p_{0}\right)} .
\end{gathered}
$$

Moreover $q>1$ implies: $\int_{E-E^{1} u^{\prime}}\left|u^{\prime}\right|^{q} \mathrm{~d} x \leqq \int_{E-E^{1} u^{\prime}}\left|u^{\prime}\right| \mathrm{d} x$, from which, using again $(1.1)^{\prime}$ we have:

(1.5) $\quad \int_{E}\left|u^{\prime}\right|^{q} \mathrm{~d} x \leqq \bar{K}(\gamma, \eta)\left[\int_{E}\left|u^{\prime}\right| \mathrm{d} x+\left.\left.|| u^{\prime}\right|^{\alpha} u^{\prime \prime}\left|{ }_{E^{\prime} u^{\prime}, p}^{p p_{0} /\left(p+p_{0}\right)}\right| u\right|_{E^{1} u^{\prime}, p_{0}} ^{p p_{0} /\left(p+p_{0}\right)}\right]$, with $\bar{K}(\gamma, \eta)=2^{1 / \eta}((\gamma+2) / \eta)^{1 / \eta}$.

From (1.5) and the second inequality of (1.2) it follows that:

$$
\left|u^{\prime}\right|_{E, q} \leqq 2^{(1 / q)+(1 / \eta q)}\left(\frac{\gamma+2}{\eta}\right)^{1 / \eta q}\left[\left.\left.|| u^{\prime}\right|^{\alpha} u^{\prime \prime}\right|_{E^{1} u^{\prime}, p} ^{1(\alpha+2)}|u|_{E_{u^{\prime}}^{\prime} p_{0}}^{1 /(\alpha+2)}+\left|u^{\prime}\right|_{E, 1}^{1 / q}\right] \text {. }
$$

Hence the assertion, remembering $(1.1)^{\prime}$. q.e.d.

Lemma 1.II. Let $\eta, \gamma ; p, p_{0}, \eta$ satisfy (1.1). Then:

$$
\left|u^{\prime}\right|_{E, q} \leqq \bar{K}\left[\left.\left.|| u^{\prime}\right|^{\alpha} u^{\prime \prime}\right|_{E^{1} u^{\prime}, p} ^{1 /(\alpha+2)}|u|_{E^{1} u^{\prime}, p_{0}}^{1 /(\alpha+2)}+\left|u^{\prime}\right|_{E, 1}^{1 / q}\right]
$$

$\forall u \in C^{2}(E)$, with $u(a)=u(b)=0$, where:

$$
q=\frac{p p_{0}(\alpha+2)}{p+p_{0}}, \bar{K}=\bar{K}(\gamma, \eta)=2^{1 / \eta}\left(\frac{\gamma+2}{\eta}\right)^{1 / \eta} .
$$

Proof. By the previous lemma, (1.6) is true for the functions $u \in C^{2}(E)$ for which $u(a)=u(b)=0$ as well as $\left|u^{\prime}(a)\right|,\left|u^{\prime}(b)\right| \leqq 1$.

Let us suppose that the last condition does not hold and that, for example; $u^{\prime}(a)>1,\left|u^{\prime}(b)\right| \leqq 1$ (the other cases can be handled similarly).

In this case the set $E_{u^{\prime}}^{1} \equiv\left\{x \in E:\left|u^{\prime}(x)\right|>1\right\}$ is not open in $R$, since the point a belongs to it. But it easy to see that: $E_{u^{\prime}}^{1}=\left[a, \beta\left[\cup \tilde{E}_{u^{\prime}}^{1} \equiv\left[a_{0}, b_{0}\left[\cup \tilde{E}_{u^{\prime}}^{1}\right.\right.\right.\right.$, where $a<\beta<b$ and $^{1}$ ) $\left.\tilde{E}_{u^{\prime}}^{1} \subseteq\right] \beta, b[$ is open in $R$.

Therefore:

$$
E_{u^{\prime}}^{1}=\left[a, \beta\left[\cup\left(\bigcup_{i \geqq 1}\right] a_{i}, b_{i}[)\right.\right.
$$

1) See p. 393. 
with

$$
u^{\prime}(x)>1 \text { in }\left[a, \beta\left[, \quad u^{\prime}(\beta)=1,\right.\right.
$$

and

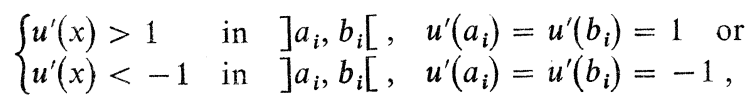

where the sets $] a_{i}, b_{i}$ [ are mutually disjoint. It is clear that (1.4) holds for the interval ]$a_{i}, b_{i}[(i \geqq 1)$.

For the interval $\left[a, \beta\left[\right.\right.$, where $u^{\prime}(x)>1, u^{\prime}(\beta)=1, u(a)=0$, we have:

$$
J_{0} \equiv \int_{a}^{\beta}\left|u^{\prime}\right|^{q} \mathrm{~d} x=\int_{a}^{\beta} u^{q-1} u^{\prime} \mathrm{d} x=
$$

$=u(\beta)-u(a)-(q-1) \int_{a}^{\beta} u^{\prime q-2} u^{\prime \prime} u \mathrm{~d} x \leqq \int_{a}^{\beta}\left|u^{\prime}\right| \mathrm{d} x+\left.\left.(q-1) \int_{a}^{\beta}|| u^{\prime}\right|^{\alpha} u^{\prime \prime}|| u^{\prime}\right|^{\sigma}|u| \mathrm{d} x$, with: $\sigma=q-2-\alpha$.

At this point, summing up (1.4) (for $i \geqq 1$ ) and (1.7) we reach a formula analogous $(1.4)^{\prime}$, with $\sum_{i=0}^{\infty} J_{i}$ instead of $\sum_{i=1}^{\infty} J_{i}$, and:

$$
E_{u^{\prime}}^{1}=\left[a, \beta\left[\cup\left(\bigcup_{i \geqq 1}\right] a_{i}, b_{i}[)=\left\{x \in E:\left|u^{\prime}(x)\right|>1\right\} .\right.\right.
$$

Hence, going on as in the lemma 1.I, we obtain (1.5) and the assertion. q.e.d.

Remark 1.I The proofs of the lemma 1:I and 1.II imply:

with:

$$
\begin{aligned}
& \int_{E}\left|u^{\prime}\right|^{q} \mathrm{~d} x \leqq \bar{K}(\gamma, \eta)\left[|| u^{\prime \alpha} u^{\prime \prime}||_{E_{u^{\prime}}, p}^{p p_{0} /\left(p+p_{0}\right)}|u|_{E_{u^{\prime}}, p_{0}}^{p p_{0} /\left(p+p_{0}\right)}+\int_{E}\left|u^{\prime}\right| \mathrm{d} x\right], \\
& \forall u \in\left\{w \in C^{2}(E): w(a)=w(b)=0\right\} \cup \\
& \cup\left\{w \in C^{2}(E):\left|w^{\prime}(a)\right|,\left|w^{\prime}(b)\right| \leqq 1\right\},
\end{aligned}
$$

$$
q=\frac{p p_{0}(\alpha+2)}{p+p_{0}}, \quad \bar{K}(\gamma, \eta)=2^{1 / \eta}\left(\frac{\gamma+2}{\eta}\right)^{1 / \eta} .
$$

Let $I$ be the interval $[0,1]$ and

$$
C_{\#}^{2}(I)=\left\{u \in C^{2}(I): u^{(k)}(0)=u^{(k)}(1), k=0,1,2\right\} .
$$

The following lemma holds:

Lemma 1.III. Let $\eta, \gamma ; p, p_{0}, \alpha$ satisfy (1.1). Then:

$$
\left|u^{\prime}\right|_{I, q} \leqq \bar{K}\left[\left.\left.|| u^{\prime}\right|^{\alpha} u^{\prime \prime}\right|_{I_{u^{\prime}}, p} ^{1 /(\alpha+2)}|u|_{I_{u}^{\prime}, p_{0}}^{1 /(\alpha+2)}+\left|u^{\prime}\right|_{I, 1}^{1 / q}\right], \quad \forall u \in C_{\#}^{2}(I)
$$

where:

$$
q=\frac{p p_{0}(\alpha+2)}{p+p_{0}}, \quad \bar{K} \equiv \bar{K}(\gamma, \eta)=2^{1 / \eta}\left(\frac{\gamma+2}{\eta}\right)^{1 / \eta} .
$$

${ }^{1}$ ) For this $u(x), \widetilde{E}_{u^{\prime}}^{1}=\emptyset$.

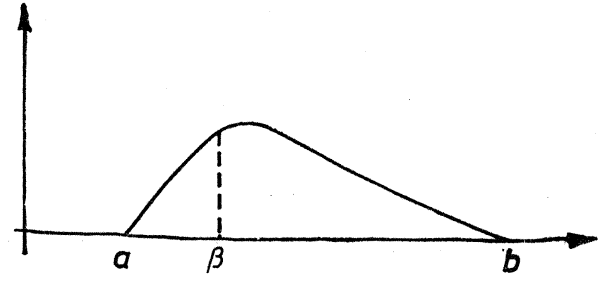


Proof. For any $u \in C_{\#}^{2}(I)$, we consider the function

which belongs to $C^{2}([0,2])$.

$$
\mathscr{U}(x)=\left\{\begin{array}{lll}
u(x) & \text { in }[0,1] \\
u(x-1) & \text { in }[1,2]
\end{array}\right.
$$

Moreover, because $u(0)=u(1)$, there exists $a \in] 0,1\left[\right.$ such that $u^{\prime}(a)=0$. Consequently, $\mathscr{U}^{\prime}(a)=\mathscr{U}^{\prime}(a+1)=0$. Thus, applying lemma $1 . I$ to the interval $E=$ $=[a, a+1]$, we get the assertion. q.e.d.

Theorem 1.I. Let $\eta, \gamma ; p, p_{0}, \alpha$ satisfy (1.1). Then:

where:

$$
\begin{gathered}
\left|u^{\prime}\right|_{E, q} \leqq \bar{K}_{1}\left[\left.\left.|| u^{\prime}\right|^{\alpha} u^{\prime \prime}\right|_{E_{u^{\prime}}, p} ^{1 / \alpha+2)}|u|_{E_{u^{\prime}}, p_{0}}^{1 /(\alpha+2)}+(\text { meas } E)^{1 / q}\right], \\
\forall u \in C^{2}(E), \quad \text { with } u(a)=u(b)=0, \\
\left|u^{\prime}\right|_{E, q} \leqq \bar{K}_{1}\left[\left.\left.|| u^{\prime}\right|^{\alpha} u^{\prime \prime}\right|_{E_{u}^{\prime} \beta_{u}, p} ^{1 /(\alpha+2)}|u|_{E, p_{0}}^{1 /(\alpha+2)}+|u|_{E, p_{0}}\right], \\
\forall u \in C^{2}(E), \text { with } u(a)=u(b)=0,
\end{gathered}
$$

$$
\begin{gathered}
E=[a, b], \quad q=\frac{p p_{0}(\alpha+2)}{p+p_{0}}, \\
\beta_{u}=|u|_{E, p_{0}}(\text { meas } E)^{-1 / q}, \quad E_{u^{\prime}}^{\beta_{u}}=\left\{x \in E:\left|u^{\prime}(x)\right|>\beta_{u}\right\}, \\
\bar{K}_{1} \equiv \bar{K}_{1}(\gamma, \eta)=2^{1+(1 / \eta)} \bar{K}^{1 / \eta} \equiv 2^{1+(1 / \eta)+\left(1 / \eta^{2}\right)}\left(\frac{\gamma+2}{\eta}\right)^{1 / \eta^{2}} .
\end{gathered}
$$

Proof. From (1.5)' of Remark 1.I, and the Hölder inequality we deduce:

$$
\begin{gathered}
\int_{E}\left|u^{\prime}\right|^{q} \mathrm{~d} x \leqq\left.\left. 2^{1 / \eta}\left(\frac{\gamma+2}{\eta}\right)^{1 / \eta}|| u^{\prime}\right|^{\alpha} u^{\prime \prime}\right|_{E_{u^{\prime}}, p} ^{p p_{0} /\left(p+p_{0}\right)}|u|_{E_{u^{\prime}}, p_{0}}^{p p_{0} /\left(p+p_{0}\right)}+ \\
+2^{1 / \eta}\left(\frac{\gamma+2}{\eta}\right)^{1 / \eta}(\text { meas } E)^{(q-1) / q}\left(\int_{E}\left|u^{\prime}\right|^{q} \mathrm{~d} x\right)^{1 / q}
\end{gathered}
$$

Hence, using the first inequality of (1.2) [with: $\left.\alpha_{1}=0, \alpha_{2}=1 / q, q_{1}=q_{2}=2\right]$ and $(1.1)^{\prime}$, we have:

$$
\begin{gathered}
\int_{E}\left|u^{\prime}\right|^{q} \mathrm{~d} x \leqq\left.\left. 2^{1+(1 / \eta)}\left(\frac{\gamma+2}{\eta}\right)^{1 / \eta}|| u^{\prime}\right|^{\alpha} u^{\prime \prime}\right|_{E_{u^{\prime}}, p} ^{p p_{0} /\left(p+p_{0}\right)}|u|_{E_{u^{\prime}}, p_{0}}^{p p_{0} /\left(p+p_{0}\right)}+ \\
+\left[2^{1+(1 / \eta)}\left(\frac{\gamma+2}{\eta}\right)^{1 / \eta}(\text { meas } E)^{(q-1) / q}\right]^{1 /(1-(1 / q))} \leqq \\
\leqq \\
{\left[2^{1+(1 / \eta)}\left(\frac{\gamma+2}{\eta}\right)^{1 / \eta}\right]^{1+1 / \eta}\left\{(\text { meas } E)+\left.\left.|| u^{\prime}\right|^{\alpha} u^{\prime \prime}\right|_{E_{u^{\prime}}, p} ^{p p_{o^{\prime}} /\left(p+p_{0}\right)}|u|_{E_{u^{\prime}}, p_{0}}^{p p_{0} /\left(p+p_{0}\right)}\right\} .}
\end{gathered}
$$

From this, (1.1)' and the second inequality of (1.2), the estimate (1.9) follows.

From (1.9) we trivially get:

$$
\begin{gathered}
\left|c u^{\prime}\right|_{E, q} \leqq \bar{K}_{1}\left[\left.\left.|| c u^{\prime}\right|^{\alpha} c u^{\prime \prime}\right|_{E_{c u^{\prime}, p}} ^{1 /(\alpha+2)}|c u|_{E, p_{0}}^{1 /(\alpha+2)}+(\text { meas } E)^{1 / q}\right], \\
\forall u \in C^{2}(E), \text { with } u(a)=u(b)=0, \quad \forall c \in R,
\end{gathered}
$$


where, clearly:

Moreover, since:

$$
E_{c u^{\prime}}^{1}=\left\{x \in E:\left|c u^{\prime}(x)\right|>1\right\}
$$

(1.9)' implies:

$$
\begin{gathered}
\forall c \in \mathbb{R}, \quad \forall A \subseteq E,\left.\left.\quad|| c u^{\prime}\right|^{\alpha} c u^{\prime \prime}\right|_{A, p} \equiv \\
\equiv\left(\int_{A}\left|c u^{\prime}(x)\right|^{\alpha p}\left|c u^{\prime \prime}(x)\right|^{p} \mathrm{~d} x\right)^{1 / p}=\left.\left.|c|^{\alpha+1}|| u^{\prime}\right|^{\alpha} u^{\prime \prime}\right|_{A, p},
\end{gathered}
$$

$$
\begin{gathered}
|c| \cdot\left|u^{\prime}\right|_{E, q} \leqq \bar{K}_{1}\left[\left.\left.|c| \cdot|| u^{\prime}\right|^{\alpha} u^{\prime \prime}\right|_{E_{u^{\prime}}^{1 / c^{\prime} \mid}, p} ^{1 /(\alpha+2)}|u|_{E, p_{0}}^{1 /(\alpha+2)}+(\text { meas } E)^{1 / q}\right], \\
\forall u \in C^{2}(E) \text { with } u(a)=u(b)=0, \quad \forall c \in R
\end{gathered}
$$

by remarking that:

$$
E_{c u^{\prime}}^{1} \equiv\left\{x \in E:\left|c u^{\prime}(x)\right|>1\right\}=\left\{x \in E:\left|u^{\prime}(x)\right|>\frac{1}{|c|}\right\} \equiv E_{u^{\prime}}^{|1 / c|} .
$$

Regarding (1.10) we observe that it is certainly true for the function $u \equiv 0$. So, we suppose $|u|_{E, p_{0}}>0$, and take:

$$
c=\beta_{u}^{-1} \equiv|u|_{E, p_{0}}^{-1}(\text { meas } E)^{1 / q}>0 .
$$

From (1.9)", written with such $a<c$, we get (1.10) and the assertion. q.e.d.

Remark 1. II. It is not clear yet if (1.10) holds, in general, (in the case $\alpha<0$ ) for any function $u \in C^{2}(E)$ regardless of the conditions in $a$ and $b$.

The fact that inequality (1.3) is not true for some $u \in C^{2}(E)$ seems to give rise to a negative answer.

In fact, for the function $u(x)=n x(n \in N)$, we have:

$$
\left.\left.|| u^{\prime}\right|^{\alpha} u^{\prime \prime}\right|_{E_{u^{\prime}, p}, p}=0, \quad\left|u^{\prime}\right|_{E, 1}^{1 / q}=n^{1 / q}(\text { meas } E)^{1 / q}, \quad\left|u^{\prime}\right|_{E, q}=n(\text { meas } E)^{1 / q} .
$$

Remark 1.II. In C. Miranda's paper, [7], the starting point is the following:

Lemma 2.II. Let $\alpha, p, p_{0}, \eta$ be real numbers satisfying:

$$
\begin{gathered}
\alpha \geqq 0, \quad p \geqq 1, \quad p_{0} \geqq 0, \\
1 \leqq q_{1} \equiv \frac{p p_{0}(\alpha+2)}{p+p_{0}} \leqq \eta(\alpha+2) .
\end{gathered}
$$

Then, for every $u \in C^{2}(] 0,1[)$ such that $u^{\prime}(x)=0$ for at least one $\left.x \in\right] 0,1[$ we have: $(2.2)_{\mathrm{M}}\left(\int_{0}^{1}\left|u^{\prime}\right|^{q_{1}} \mathrm{~d} x\right)^{1 / q_{1}} \leqq K_{0}\left(\int_{0}^{1}\left|u^{\prime}\right|^{\alpha p}\left|u^{\prime \prime}\right|^{p} \mathrm{~d} x^{(1 / p)(1 /(\alpha+2))}\left(\int_{0}^{1}|u|^{p_{0}} \mathrm{~d} x\right)^{\left(1 / p_{0}\right)(1 /(\alpha+2))}\right.$, whenever the right-hand side is finite, with $K_{0}$ depending only on $\eta$.

When, in particular, $u \in C^{2}([0,1])$ (which is the starting point in the proof of the lemma 2.II), formula $(2.2)_{M}$ is always meaningful, since the right-hand side of $(2.2)_{M}$ is always finite.

If we try to extend lemma 2 .II to the case $\alpha<0$, even remaining in the class $C^{2}([0,1])$, it is necessary to substitute $(2.2)_{\mathrm{M}}$ with:

$$
\left(\int_{0}^{1}\left|u^{\prime}\right|^{q_{1}} \mathrm{~d} x\right)^{1 / q_{1}} \leqq K_{0}\left(\int_{\left\{x: u^{\prime}(x) \neq 0\right\}}\left|u^{\prime}\right|^{\alpha p}\left|u^{\prime \prime}\right|^{p} \mathrm{~d} x\right)^{(1 / p)(1 /(x+2))}\left(\int_{0}^{1}|u|^{p_{0}} \mathrm{~d} x\right)^{\left(1 / p_{0}\right)(1 /(\alpha+2))}
$$


$\forall u \in C^{2}([0,1])$ such that $u^{\prime}=0$ in at least one point of $[0,1]$. Immediately we find out that $\alpha(<0)$ cannot be arbitrary, if we want to make finite the right-hand side.

For instance, if $p=1, u(x)=x^{\beta}, \beta \geqq 2\left(\Rightarrow u^{\prime}(0)=0\right)$ the right-hand side will be finite only if $\alpha>-1$. On the other side, the condition $\alpha>-1$ is essential if we want to write (with $\alpha<0$ ) the formula (2.4) in the proof of the lemma 2.II ([7], page 4).

Actually there exist negative $\alpha$ 's, arbitrarily close to zero and functions $u \in C^{2}([0,1])$ (with $u^{\prime}=0$ in a point of $[0,1]$ ) such that the right-hand side of $(2.2)^{\prime}$ is not finite, so that $(2.2)^{\prime}$ does not give any information. It is enough to consider, for example:

(i) $p=2, \quad u(x)=\psi(x) \int_{0}^{x} t^{k+2} \operatorname{sen}^{2}\left(t^{-k}\right) \mathrm{d} t, \quad \alpha=\frac{-3}{(k+2) 2}$

$$
\text { with }\left\{\begin{array}{l}
k \text { integer } \geqq 1 \\
\psi \in C_{0}^{\infty}(]-1,1[), \quad \psi \equiv 1 \text { for }|x| \leqq \frac{1}{2} .
\end{array}\right.
$$

In fact, it is clear that;

which implies: $u \in C^{2}([0,1])$.

$$
\begin{gathered}
\forall x \in] 0, \frac{1}{2}\left[,\left\{\begin{aligned}
u^{\prime}(x)= & x^{k+2} \operatorname{sen}^{2}\left(x^{-k}\right) \\
u^{\prime \prime}(x)= & x \operatorname{sen}\left(x^{-k}\right)\left[(k+2) x^{k} \operatorname{sen}\left(x^{-k}\right)-2 k \cos \left(x^{-k}\right)\right] \\
& u^{\prime}(0)=\lim _{x \rightarrow 0^{+}} u^{\prime}(x)=0, \\
& u^{\prime \prime}(0)=\lim _{x \rightarrow 0^{+}} u^{\prime \prime}(x)=0,
\end{aligned}\right.\right.
\end{gathered}
$$

Then, $\forall n \in N, n>0$ and $\forall k \in N, k \geqq 1$, we have:

(iii) $\left.\forall x \in I_{n} \equiv\right]\left(\frac{1}{4} \pi+2 n \pi\right)^{-1 / k},\left(\frac{1}{6} \pi+2 n \pi\right)^{-1 / k}[$,

$$
\frac{1}{2} \leqq\left|\operatorname{sen}\left(x^{-k}\right)\right| \leqq \sqrt{ } 2 / 2 \leqq 2 k\left|\cos \left(x^{-k}\right)\right|-(k+2) x^{k}\left|\operatorname{sen}\left(x^{-k}\right)\right| \text {. }
$$

From (i), (ii), (iii) we obtain:

(iv)

$$
\begin{gathered}
\forall x \in I_{n}, \quad\left|u^{\prime}(x)\right|^{\alpha p}\left|u^{\prime \prime}(x)\right|^{p} \equiv \\
\equiv\left|u^{\prime}(x)\right|^{-3 /(k+2)}\left|u^{\prime \prime}(x)\right|^{2}=\left(x^{-3}\left|\operatorname{sen}\left(x^{-k}\right)\right|^{-6 /(k+2)}\right) . \\
.\left(x^{2}\left|\operatorname{sen}\left(x^{-k}\right)\right|^{2}\left|(k+2) x^{k} \operatorname{sen}\left(x^{-k}\right)-2 k \cos \left(x^{-k}\right)\right|^{2}\right) \geqq 1 / 8 x
\end{gathered}
$$

for every integer $k \geqq 1$ and $n>2^{k-1} / \pi$.

Consequently:

$$
J_{n} \equiv \int_{I_{n}}\left|u^{\prime}\right|^{\alpha p}\left|u^{\prime \prime}\right|^{p} \mathrm{~d} x \geqq \frac{1}{8} \int_{I_{n}} \frac{\mathrm{d} x}{x}=\frac{1}{8 k} \log \frac{3+24 n}{2+24 n},
$$

from which we get:

$$
\begin{aligned}
& \text { (V) } \int_{\left\{x: u^{\prime}(x) \neq 0\right\}}\left|u^{\prime}(x)\right|^{\alpha p}\left|u^{\prime \prime}(x)\right|^{p} \mathrm{~d} x \geqq \sum_{n>2^{k} / 2 \pi} J_{n} \geqq \sum_{n>2^{k} / 2 \pi} \frac{1}{8 k} \log \frac{3+24 n}{2+24 n}= \\
& =\frac{1}{8 k} \log \prod_{n>2^{k} / 2 \pi}\left(1+\frac{1}{2+24 n}\right)=+\infty \text {. }
\end{aligned}
$$


Therefore, with this choice of $u(x), \alpha$ and $p=2$, the right-hand side of $(2.2)^{\prime}$ is not finite, whatever $p_{0}$ is. Thus, if we an estimate similar to $(2.2)_{M}$ for $\alpha<0$, which is meaningful for a reasonably large class of functions $u \in C^{2}([0,1])$, it is necessary to restrict the set $I$ over which the integral of $\left|u^{\prime}\right|^{\alpha p}\left|u^{\prime \prime}\right|^{p}$ is taken. In doing this we should be careful to restrict $I$ so much that:

$$
\int_{I}\left|u^{\prime}\right|^{\alpha p}\left|u^{\prime \prime}\right|^{p} \mathrm{~d} x<+\infty
$$

but so little that it would still be possible to estimate the left-hand side of $(2.2)^{\prime}$ by the above integral. This is exactly what we manage to do in Theorem 1.I, even if under hypotheses (1.1) and for the $u$ 's in $C^{2}([a, b])$ which are zero in $a$ and $b$.

For example, the function $u(x)$ defined in (i) satisfies this condition in $[a, b] \equiv$ $\equiv[0,1]$. Moreover, since for $k \geqq 1$, the numbers:

$$
\left.p=2, \quad \eta=\frac{1}{4}, \quad \alpha=-3 /(2 k+4) \in\right] \frac{5}{8}-2,0[
$$

verify $(1.1)_{\infty}$, we know that the numbers $\eta=\frac{1}{4}, \gamma=0, p=2, \alpha=-3 /(2 k+4)$ and

$$
p_{0} \geqq \bar{p}>\max \left\{2, \frac{(1+\eta) p}{p(\alpha+2)-1-\eta}\right\} \equiv 2
$$

satisfy (1.1); therefore (1.10) and (1.9) holds for the function $u(x)$ of (i).

Consequently these formula's give real information on $u(x)$ whereas $(2.2)^{\prime}$ cannot say anything since the right-hand side is not finite.

Remark 1.IV. Let $f$ be a continuous function in $E=[a, b]$. Then for any measurable subset $B \subseteq E$, the following mean values are defined by:

$$
\begin{gathered}
m_{B}(f)=\frac{1}{\operatorname{meas} B} \int_{B} f(x) \mathrm{d} x, \quad M_{r, B}(f)=\left(\frac{1}{\operatorname{meas} B} \int_{B}|f|^{r} \mathrm{~d} x\right)^{1 / \boldsymbol{r}}, \\
\mu_{\boldsymbol{r}, B}(f)=\left(\frac{1}{\text { meas } B} \int_{B}\left|f-m_{B}(f)\right|^{\boldsymbol{r}} \mathrm{d} x\right)^{1 / \boldsymbol{r}}
\end{gathered}
$$

where $r$ is a positive real number.

For any $f \in C^{1}(E)$, we define the following function:

$$
u(x)=\int_{a}^{x} f(t) \mathrm{d} t-\frac{x-a}{b-a} \int_{a}^{b} f(t) \mathrm{d} t, \quad x \in E \equiv[a, b],
$$

which satisfies the hypotheses of Theorem 1.I $\left(u \in C^{2}(E)\right)$ since $u(a)=u(b)=0$ and $u^{\prime}(x)=f(x)-m_{E}(f), u^{\prime \prime}(x)=f^{\prime}(x) \in C^{0}(E)$.

Then, if $\alpha<0, p, p_{0}, \eta$ verify (together with $\gamma=0$ ) condition (1.1), writing (1.9) for this $u$, we have:

$$
\left(\int_{E}\left|f-m_{E}(f)\right|^{q} \mathrm{~d} x\right)^{1 / q} \leqq
$$

$$
\leqq \bar{K}_{1}\left[(\text { meas } E)^{1 / q}+\left(\int_{E_{u^{\prime}}{ }^{1}}\left|u^{\prime}\right|^{p x}\left|u^{\prime \prime}\right|^{p} \mathrm{~d} x\right)^{1 / p(\alpha+2)}\left(\int_{E_{u^{\prime}}{ }^{1}}|u|^{p_{0}} \mathrm{~d} x\right)^{1 /\left(p_{0}(\alpha+2)\right)}\right],
$$

where, obviously, $E_{u^{\prime}}^{1} \equiv E^{1}=\left\{x \in E:\left|f(x)-m_{E}(f)\right|>1\right\}$. Considering the expres- 
sion of $u(x)$ we get:

From (1.12) it follows:

$$
\left(\int_{E^{1}}|u|^{p_{0}} \mathrm{~d} x\right)^{1 / p_{0}} \leqq 2(b-a)^{\left(p_{0}-1\right) / p_{0}}\left(\text { meas } E^{1}\right)^{1 / p_{0}}\left(\int_{E}|f|^{p_{0}} \mathrm{~d} x\right)^{1 / p_{0}} .
$$

$$
\begin{gathered}
\left(\int_{E}\left|f(x)-m_{E}(f)\right|^{q} \mathrm{~d} x\right)^{1 / q} \leqq \\
\leqq \bar{K}_{1}\left[(\text { meas } E)^{1 / q}+2^{1 /(\alpha+2)}(\text { meas } E)^{\left(p_{0}-1\right) / p_{0}(\alpha+2)}\left(\text { meas } E^{1}\right)^{1 / p_{0}(\alpha+2)} .\right. \\
\left..\left(\int_{E}|f|^{p_{0}} \mathrm{~d} x\right)^{1 /\left(p_{0}(\alpha+2)\right)}\left(\int_{E^{1}}\left|f^{\prime}\right|^{p}\left|f-m_{E}(f)\right|^{p z} \mathrm{~d} x\right)^{1 / p(\alpha+2)}\right] .
\end{gathered}
$$

Finally, dividing by (meas $E)^{1 / q}$ and remembering that

$(1.12)^{\prime}$ implies:

$$
\frac{1}{q}=\frac{p+p_{0}}{p p_{0}(\alpha+2)}=\frac{1}{p(\alpha+2)}+\frac{1}{p_{0}(\alpha+2)},
$$

$$
\mu_{q, E}(f) \leqq \bar{K}_{1}+
$$

$+\bar{K}_{1} 2^{1 /(\alpha+2)}\left(\operatorname{meas} E^{1}\right)^{1 / q}(\operatorname{meas} E)^{1 /(\alpha+2)-(1 / q)} M_{p_{0}, E}^{1 /(\alpha-2)}(f) M_{p_{0}, E^{1}}^{1 /(\alpha+2)}\left(\frac{\left|f^{\prime}\right|}{\left|f-m_{E}(f)\right|^{|\alpha|}}\right)$

for every $f \in C^{1}(E)$.

\section{INEQUALITIES FOR FUNCTIONS OF SEVERAL VARIABLES}

For each $i \in\{1, \ldots, n\}$ we consider the one-to-one map:

$$
\varphi_{i}:(y, t) \equiv\left(\left(y_{1}, \ldots, y_{n-1}\right), t\right) \in \mathbb{R}^{n-1} \times \mathbb{R} \rightarrow x \equiv\left(x_{1}, \ldots, x_{n}\right) \in \mathbb{R}^{n}
$$

defined by the equations:

$$
x_{j}=y_{j} \text { for } j<i, \quad x_{i}=t, \quad x_{j}=y_{j-1} \text { for } j>i .
$$

We write: $x \equiv \varphi_{i}(y, t)=(y ; t)_{i}$, or $x=(y ; t)$ whenever $i$ is fixed. Obviously we have:

$$
\frac{\partial(x)}{\partial(y, t)} \equiv \frac{\partial(y ; t)_{i}}{\partial(y, t)}=(-1)^{n-i}
$$

Let $\Omega$ be a bounded convex open set of $R^{n}$ and set:

$$
\begin{gathered}
\forall y \in \mathbb{R}^{n-1}, \quad \Omega^{i}(y)=\left\{t \in \mathbb{R}: x \equiv \varphi_{i}(y, t) \in \bar{\Omega}\right\} \equiv\left[a^{i}(y), b^{i}(y)\right] \text { if } \Omega^{i}(y) \neq \emptyset, \\
\Omega^{i}=\left\{y \in \mathbb{R}^{n-1}: \Omega^{i}(y) \neq \emptyset\right\} .
\end{gathered}
$$

If $u(x)$ is a summable function in $\bar{\Omega}$, evidently:

$$
\int_{\bar{\Omega}} u(x) \mathrm{d} x=\int_{\left\{(y, t): \varphi_{i}(y, t) \in \bar{\Omega}\right\}} u\left(\varphi_{i}(y, t)\right)\left|\frac{\partial(x)}{\partial(y, t)}\right| \mathrm{d} y \mathrm{~d} t=\int_{\Omega^{i}} \mathrm{~d} y \int_{\Omega^{i}(y)} u(y ; t) \mathrm{d} t .
$$

Theorem 2.I. Let $\eta, \gamma ; p, p_{0}, \alpha$ satisfy (1.1) and $\Omega$ be a bounded convex open set in $R^{n}$. Ther we have:

$$
\begin{gathered}
\left|D_{x_{i}} u\right|_{\Omega, q} \leqq \bar{K}\left[\left.\left.|| D_{x_{i}} u\right|^{\alpha} D_{x_{i}}^{2} u\right|_{\Omega_{i u^{1}, p}} ^{1 /(\alpha+2)}|u|_{\left.\Omega_{i u^{1}, p_{0}}^{1 /(\alpha+2)}+\left|D_{x_{i}} u\right|_{\Omega, 1}^{1 / q}\right]}^{1 / \alpha}, \quad \text { with }\left.u\right|_{\partial \Omega}=0\right. \\
\forall u \in C^{2}(\bar{\Omega}) \text {, }
\end{gathered}
$$


where: $i=1, \ldots, n ; q=p p_{0}(\alpha+2) /\left(p+p_{0}\right)$;

Moreover:

$$
\begin{gathered}
\bar{K} \equiv \bar{K}(\gamma, \eta)=2^{1 / \eta}\left(\frac{\gamma+2}{\eta}\right)^{1 / \eta} \\
\Omega_{i, u}^{1}=\left\{x \in \bar{\Omega}:\left|D_{x_{i}} u(x)\right|>1\right\} .
\end{gathered}
$$

where:

$$
\begin{gathered}
\left|D_{x_{i}} u\right|_{\Omega, q} \leqq \bar{K}_{1}\left[\left.\left.|| D_{x_{i}} u\right|^{\alpha} D_{x_{i}}^{2} u\right|_{\Omega_{u^{1}, p}} ^{1 /(\alpha+2)}|u|_{\Omega_{i u^{1}, p_{0}}^{1 /(\alpha+2)}}^{\left.1 /(\text { meas } \Omega)^{1 / q}\right]}\right. \\
\forall u \in C^{2}(\bar{\Omega}) \text { with }\left.u\right|_{\partial \Omega}=0
\end{gathered}
$$

$$
\bar{K}_{1} \equiv \bar{K}_{1}(\gamma, \eta)=2^{1+(1 / \eta)+\left(1 / \eta^{2}\right)}\left(\frac{\gamma+2}{\eta}\right)^{1 / \eta^{2}} .
$$

Proof. Fix $i \in\{1, \ldots, n\}$. Since $\forall y \in \Omega^{i}$ and $\forall u \in C^{2}(\bar{\Omega})$ with $\left.u\right|_{\partial \Omega}=0$, the function $u(y ; \cdot) \in C^{2}\left(\Omega^{i}(y)\right), u\left(y ; a^{i}(y)\right)=u\left(y ; b^{i}(y)\right)=0$, we can write for it formula $(1.5)^{\prime}$ of Remark 1.I (with $E=\Omega^{i}(y) \equiv\left[a^{i}(y), b^{i}(y)\right]$ and $E_{u^{\prime}}^{1}=\left\{t \in \Omega^{i}(y):\left|D_{x_{i}} u(y ; t)\right|>1\right\}$ ) and then take the integral, with respect to $y$, over $\Omega^{i}$. Applying Hölder's inequality, we obtain:

$$
\begin{aligned}
& \int_{\Omega}\left|D_{x_{i}} u(x)\right|^{q} \mathrm{~d} x \equiv \int_{\Omega^{i}} \mathrm{~d} y \int_{\Omega^{i}(y)}\left|D_{x_{i}} u(y ; t)\right|^{q} \mathrm{~d} t \leqq \\
& \leqq \bar{K} \int_{\Omega^{i}}\left(\int_{\left\{t \in \Omega^{i}(y):\left|D_{x_{i}} k(y ; t)\right|>1\right\}}\left|D_{x_{i}} u(y ; t)\right|^{p \alpha}\left|D_{x_{i}}^{2} u(y ; t)\right|^{p} \mathrm{~d} t\right)^{p_{0} /\left(p+p_{0}\right)} \text {. } \\
& \text {. }\left(\int_{\left\{t \in \Omega^{i}(y):\left|D_{x_{i}} u(y ; t)\right|>1\right\}}|u(y ; t)|^{p_{0}} \mathrm{~d} t\right)^{p /\left(p+p_{0}\right)} \mathrm{d} y+ \\
& +\bar{K} \int_{\Omega^{i}}\left(\int_{\Omega^{i}(y)}\left|D_{x_{i}} u(y ; t)\right| \mathrm{d} t\right) \mathrm{d} y \leqq \bar{K} \int_{\Omega}\left|D_{x_{i}} u(x)\right| \mathrm{d} x+ \\
& +\bar{K}\left(\int_{\Omega^{i}} \mathrm{~d} y \int_{\left\{t \in \Omega^{i}(y):\left|D_{x_{i}} u(y ; t)\right|>1\right\}}\left|D_{x_{i}} u(y ; t)\right|^{p \alpha}\left|D_{x_{i}}^{2} u(y ; t)\right|^{p} \mathrm{~d} t\right)^{p_{0} /\left(p+p_{0}\right)} \text {. } \\
& \text {. }\left(\int_{\Omega^{i}} \mathrm{~d} y \int_{\left\{t:\left|D_{x_{i}} u(y ; t)\right|>1\right\}}|u(y ; t)|^{p_{0}} \mathrm{~d} t\right)^{\left.p / p+p_{0}\right)} \equiv \\
& \equiv \bar{K}\left[\left|D_{x_{i}} u\right|_{\Omega, 1}+\left.\left.|| D_{x_{i}} u\right|^{\alpha} D_{k_{i}}^{2} u\right|_{\Omega_{i, u^{1}, p}} ^{p p_{0} /\left(p+p_{0}\right)}|u|_{\Omega_{i, u^{1}, p_{0}}}^{p p_{0} /\left(p+p_{0}\right)}\right] \text {. }
\end{aligned}
$$

From this the estimate (2.1) follows taking the power with exponent $1 / q$ and remembering the second inequality of $(1.2)$ and $(1.1)^{\prime}$. In order to have $(2.1)^{\prime}$, first of all we write $(1.11)^{\prime}$ for $u(y ; \cdot)$ and then take the integral over $\Omega^{i}$ with respect to $y$ :

$$
\begin{gathered}
\int_{\Omega}\left|D_{x_{i}} u(x)\right|^{q} \mathrm{~d} x \equiv \int_{\Omega^{i}} \mathrm{~d} y \int_{\Omega^{i}(y)}\left|D_{x_{i}} u(y ; t)\right|^{q} \mathrm{~d} t \leqq \\
\leqq\left(2^{1+(1 / \eta)}\left(\frac{\gamma+2}{\eta}\right)^{1 / \eta}\right)^{1+(1 / \eta)}\left[\int_{\Omega^{i}}\left(\text { meas } \Omega^{i}(y)\right) \mathrm{d} y+\right. \\
+\int_{\Omega^{i}}\left(\int_{\left\{t \in \Omega^{i}(y):\left|D_{x_{i}} u(y ; t)\right|>1\right\}}\left|D_{x_{i}} u(y ; t)\right|^{p_{\alpha}}\left|D_{x_{i}}^{2} u(y ; t)\right|^{p} \mathrm{~d} t\right)^{p_{0} /\left(p+p_{0}\right)} . \\
\left.\cdot\left(\int_{\left\{t:\left|D_{x_{i}} u(y ; t)\right|>1\right\}}|u(y ; t)|^{p_{0}} \mathrm{~d} t\right)^{p_{0} /\left(p+p_{0}\right)} \mathrm{d} y\right] \leqq \\
\leqq\left(2^{1+(1 / \eta)}\left(\frac{\gamma+2}{\eta}\right)^{1 / \eta}\right)^{1+(1 / \eta)}\left[\operatorname{meas} \Omega+\left(\int_{\Omega_{i, u^{1}}}\left|D_{x_{i}} u\right|^{p \alpha}\left|D_{x_{i}}^{2} u\right|^{p} \mathrm{~d} x\right)^{p_{0} /\left(p+p_{0}\right)} .\right. \\
\left.\cdot\left(\int_{\Omega_{i, u}}|u|^{p_{0}} \mathrm{~d} x\right)^{p /\left(p+p_{0}\right)}\right] .
\end{gathered}
$$

Here we have also applied Hölder's inequality. 
Hence, taking the power with exponent $1 / q$ and using the second inequality of (1.2) and (1.1)' we get $(2.1)^{\prime}$ and the assertion. q.e.d.

Corollary 2.I. Let $\eta, \gamma ; p, p_{0}, \alpha$ satisfy (1.1) with $\gamma=0, \alpha<0$. Let $\Omega$ be a bounded convex open set in $R^{n}$. Then we have:

$$
\begin{array}{r}
\text { (2.3) }\left|D_{x_{i}} u\right|_{\Omega, q} \leqq \bar{K}_{2}\left[\left|\left(1+\left|D_{x_{i}} u\right|\right)^{\alpha} D_{x_{i}}^{2} u\right|_{\Omega_{i, u^{1}, p}}^{1 /(\alpha+2)}|u|_{\Omega_{i, u^{1}, p_{0}}}^{1 /(\alpha+2)}+\left|D_{x_{i}} u\right|_{\Omega, 1}^{1 / \alpha}\right], \\
\forall u \in C^{2}(\bar{\Omega}), \text { with }\left.u\right|_{\partial \Omega}=0 ; \\
(2.3)^{\prime} \quad\left|D_{x_{i}} u\right|_{\Omega, q_{2}} \leqq \bar{K}_{2}\left[\left|\left(1+\left(D_{x_{i}} u\right)^{2}\right)^{\alpha / 2} D_{x_{i}}^{2} u\right|_{\Omega_{i, u^{1}, 2}}^{1 /(\alpha+2)}|u|_{\Omega_{i, u^{1}, p_{0}}}^{1 /(\alpha+2)}+\left|D_{x_{i}} u\right|_{\Omega, 1}^{1 / q_{2}}\right], \\
\forall u \in C^{2}(\bar{\Omega}) \text { with }\left.u\right|_{\partial \Omega}=0,
\end{array}
$$

where:

$$
\begin{gathered}
i=1, \ldots, n ; \quad q=\frac{p p_{0}(\alpha+2)}{p+p_{0}} ; \quad q_{2}=\frac{2 p_{0}(\alpha+2)}{2+p_{0}} ; \\
\Omega_{i, u}^{1}=\left\{x \in \Omega:\left|D_{x_{i}} u(x)\right|>1\right\} ; \\
\bar{K}_{2} \equiv \bar{K}_{2}(\eta)=2^{2 / \eta(1+\eta)} \bar{K}(0, \eta) \equiv 2^{(2 ; n(1+\eta))+(1 / \eta)}(2 / \eta)^{1 / \eta}
\end{gathered}
$$

Proof. Since $0>\alpha>-2,\left|D_{x_{i}} u(x)\right|>1$ in $\Omega_{i, u}^{1}$, we have:

$$
\begin{gathered}
\left.\left.|| D_{x_{i}} u\right|^{\alpha} D_{x_{i}}^{2} u\right|_{\Omega_{i, u^{1}, p}} ^{1 /(\alpha+2)} \equiv\left(\int_{\Omega_{i, u^{1}}} \frac{\left|D_{x_{i}}^{2} u\right|^{p} \mathrm{~d} x}{\left|D_{x_{i}} u\right|^{p|\alpha|}}\right)^{1 / p(\alpha+2)}= \\
=2^{|\alpha| /(\alpha+2)}\left(\int_{\Omega_{i, u^{1}}}\left(2\left|D_{x_{i}} u\right|\right)^{-p|\alpha|}\left|D_{x_{i}}^{2} u\right|^{p} \mathrm{~d} x^{1 / p(\alpha+2)} \leqq\right. \\
\leqq 2^{|\alpha| /(\alpha+2)}\left(\int_{\Omega_{i, u^{1}}}\left(1+\left|D_{x_{i}} u\right|\right)^{p \alpha}\left|D_{x_{i}}^{2} u\right|^{p} \mathrm{~d} x\right)^{1 / p(\alpha+2)}, \\
\frac{|\alpha|}{\alpha+2}<\frac{2}{\eta(1+\eta)},
\end{gathered}
$$

because, by (1.1): $-|\alpha|=\alpha>\eta(1+\eta)-2$ and hence:

$$
|\alpha|<2, \quad \alpha+2>\eta(1+\eta) \text {. }
$$

From (2.4) and (2.1) we obtain (2.3), from which, for $p=2,(2.3)^{\prime}$ follows. q.e.d.

Corollary 2.II. Let $\eta, \gamma ; p, p_{0}, \alpha$ satisfy (1.1) with $\gamma=0, \alpha<0$. Let $\Omega$ be a bounded convex open set in $R^{n}$. Then we have:

$$
\begin{gathered}
\left|D_{x_{i}} u\right|_{\Omega, q} \leqq \bar{K}_{3}\left[\left|\left(1+\left|D_{x_{i}} u\right|\right)^{\alpha} D_{x_{i}}^{2} u\right|_{\Omega_{i, u^{1}, p}}^{1 /(\alpha+2)}|u|_{\Omega_{i, u^{1}, p_{0}}}^{1 /(\alpha+2)}+(\text { meas } \Omega)^{1 / q}\right], \\
\forall u \in C^{2}(\bar{\Omega}) \text { with }\left.u\right|_{\partial \Omega}=0, \\
(2.5)^{\prime}\left|D_{x_{i}} u\right|_{\Omega, q_{2}} \leqq \bar{K}_{3}\left[\left|\left(1+\left(D_{x_{i}} u\right)^{2}\right)^{\alpha / 2} D_{x_{i}}^{2} u\right|_{\Omega_{i, u^{1}, 2}}^{1 /(\alpha+2)}|u|_{\Omega_{i, u^{1}, p_{0}}}^{1 /(\alpha+2)}+(\text { meas } \Omega)^{1 / q_{2}}\right], \\
\forall u \in C^{2}(\bar{\Omega}) \text { with }\left.u\right|_{\hat{o} \Omega}=0,
\end{gathered}
$$

where: $i=1, \ldots, n ; q, q_{2}, \Omega_{i, u}^{1}$ as in the previous corollary and:

$$
\bar{K}_{3} \equiv \bar{K}_{3}(\eta)=2^{2 / \eta(1+\eta)} \bar{K}_{1}(0, \eta) \equiv 2^{(2 / \eta(1+\eta))+1+(1 / \eta)+\left(1 / \eta^{2}\right)}(2 / \eta)^{1 / \eta^{2}} .
$$

Proof. The inequality (2.5) follows immediately from (2.4) and (2.1)'. From it, for $p=2$, we get $(2.5)^{\prime}$. q.e.d. 
Corollary 2.III. Let $\alpha, \eta, p$ be three real numbers verifying $(1.1)_{\infty}$ and $\Omega$ a bounded convex open set in $R^{n}$. Then we have:

$$
\begin{gathered}
(2.6)\left|D_{x_{i}} u\right|_{\Omega, p(\alpha+2)} \leqq \bar{K}_{2}\left[\left|\left(1+\left|D_{x_{i}} u\right|\right)^{\alpha} D_{x_{i}}^{2} u\right|_{\Omega_{i, u^{1}, p}}^{1 /(\alpha+2)}\left(\sup _{\Omega}|u|\right)^{1 /(\alpha+2)}+\left|D_{x_{i}} u\right|_{\Omega, 1}^{1 /(\alpha+2)}\right], \\
\forall u \in C^{2}(\bar{\Omega}) \text { with }\left.u\right|_{\partial \Omega}=0, \\
(2.6)^{\prime}\left|D_{x_{i}} u\right|_{\Omega, 4+2 \alpha} \leqq \bar{K}_{2}\left[\left|\left(1+\left(D_{x_{i}} u\right)^{2}\right)^{\alpha / 2} D_{x_{i}}^{2} u\right|_{\Omega_{i, u^{1}, 2}}^{1 /(\alpha+2)}\left(\sup _{\Omega}|u|\right)^{1 /(\alpha+2)}+\left|D_{x_{i}} u\right|_{\Omega, 1}^{1 /(4+2)}\right], \\
\forall u \in C^{2}(\bar{\Omega}) \text { with }\left.u\right|_{\partial \Omega}=0,
\end{gathered}
$$

where:

$$
\begin{gathered}
i=1, \ldots, n ; \quad \Omega_{i, u}^{1}=\left\{x \in \Omega:\left|D_{x_{i}} u(x)\right|>1\right\} ; \\
\bar{K}_{2}=2^{(2 / \eta(1+\eta))+(1 / \eta)}(2 / \eta)^{1 / \eta}
\end{gathered}
$$

Moreover we have:

$\left.\left|D_{x_{i}} u\right|_{\Omega, p(\alpha+2)} \leqq \bar{K}_{3}\left[\left|\left(1+\left|D_{x_{i}} u\right|\right)^{\alpha} D_{x_{i}}^{2} u\right|_{\Omega_{i, u^{1}, p}}^{1 /(\alpha+2)} \sup _{\Omega}|u|\right)^{1 /(\alpha+2)}+(\text { meas } \Omega)^{1 / p(\alpha+2)}\right]$,

$$
\forall u \in C^{2}(\bar{\Omega}) \text { with }\left.u\right|_{\partial \Omega}=0,
$$

$$
\left|D_{x_{i}} u\right|_{\Omega, 4+2 \alpha} \leqq \bar{K}_{3}\left[\left|\left(1+\left(D_{x_{i}} u\right)^{2}\right)^{\alpha / 2} D_{x_{i}}^{2} u\right|_{\Omega_{i, u^{1}, 2}}^{1 /(\alpha+2)}\left(\sup _{\Omega}|u|\right)^{1 /(\alpha+2)}+(\text { meas } \Omega)^{1 /(4+2 \alpha)}\right] \text {, }
$$

where:

$$
\forall u \in C^{2}(\bar{\Omega}) \text { with }\left.u\right|_{\partial \Omega}=0
$$

Proof. As we know, for

$$
p_{0} \geqq \bar{p}>\max \left\{\frac{p}{p-1}, \frac{(1+\eta) p}{p(\alpha+2)-1-\eta}\right\}
$$

the numbers $\alpha, \eta, p, p_{0}, \gamma=0$ satisfy (1.1) (with $\alpha<0$ ). So we can write (2.3) for $p_{0} \geqq \bar{p}$.

Since the number $\bar{K}_{2} \equiv \bar{K}_{2}(\eta)$ does not depend on $p_{0}$, and

$$
\lim _{p_{0} \rightarrow+\infty} q^{-1} \equiv \lim _{p_{0} \rightarrow+\infty} \frac{p+p_{0}}{p p_{0}(\alpha+2)}=\frac{1}{p(\alpha+2)}<\frac{1}{1+\eta},
$$

taking the limit in (2.3) for $p_{0} \rightarrow+\infty$, we get (2.6). In the same way (2.6) follows from $(2.3)^{\prime}$.

But also $\bar{K}_{3}$ does not depend on $p_{0}$; so $(2.7)$ follows from $(2.5)$ and $(2.7)^{\prime}$ from $(2.5)^{\prime}$, for $p_{0} \rightarrow+\infty$. q.e.d.

Theorem 2.II. Let $\alpha, \eta, p$ satisfy $(1.1)_{\infty}$ with $\left.p=2, \alpha \in\right]-1,0[$. Let $\Omega$ be a bounded convex open set in $R^{n}$. Then we have:

$$
\begin{gathered}
\int_{\Omega}\left|D_{x_{i}}^{2} u\right|^{2+\alpha} \mathrm{d} x \leqq 2^{(1+(\alpha / 2))|\alpha|}\left[\int_{\Omega}\left(1+\left(D_{x_{i}} u\right)^{2}\right)^{\alpha}\left(D_{x_{i}}^{2} u\right)^{2} \mathrm{~d} x\right]^{1+(\alpha / 2)} \\
\cdot\left\{(\operatorname{meas} \Omega)^{|\alpha| / 2}+\bar{K}_{3}^{(2+\alpha)|\alpha|}\left[(\operatorname{meas} \Omega)^{1 /(4+2 \alpha)}+\right.\right. \\
\left.\left.+\left(\sup _{\Omega}|u|\right)^{1 /(\alpha+2)}\left(\int_{\Omega}\left(1+\left(D_{x_{i}} u\right)^{2}\right)^{\alpha}\left(D_{x_{i}}^{2} u\right)^{2} \mathrm{~d} x\right)^{1 /(4+2 \alpha)}\right]^{(2+\alpha)|\alpha|}\right\} \\
\forall u \in C^{2}(\bar{\Omega}) \text { with }\left.u\right|_{\partial \Omega}=0
\end{gathered}
$$


where:

$$
i=1, \ldots, n ; \bar{K}_{3}=2^{(2 / \eta(1+\eta))+1+(1 / \eta)+\left(1 / \eta^{2}\right)}(2 / \eta)^{1 / \eta^{2}} .
$$

Proof. Set:

$$
\varrho=2+\alpha \in] 1,2\left[, \quad \beta=\frac{1}{2} \varrho|\alpha| \equiv\left(1+\frac{\alpha}{2}\right)|\alpha|>0,\right.
$$

from which:

$$
\frac{2 \beta}{\varrho}=|\alpha| \equiv-\alpha, \frac{4 \beta}{2-\varrho}=4+2 \alpha\left(\Rightarrow \frac{2 \beta}{2-\varrho}>1\right) .
$$

Thus using Hölder and Minkowski's inequalities and the second inequality of (1.2), we obtain:

$$
\begin{gathered}
\int_{\Omega}\left|D_{x_{i}}^{2} u\right|^{2+\alpha} \mathrm{d} x \leqq\left(\int_{\Omega}\left(1+\left(D_{x_{i}} u\right)^{2}\right)^{-2 \beta / \varrho}\left(D_{x_{i}}^{2} u\right)^{2} \mathrm{~d} x\right)^{\varrho / 2} . \\
\cdot\left(\int_{\Omega}\left(1+\left(D_{x_{i}} u\right)^{2}\right)^{2 \beta /(2-\varrho)} \mathrm{d} x\right)^{(2-\varrho) / 2} \leqq\left(\int_{\Omega}\left(1+\left(D_{x_{i}} u\right)^{2}\right)^{\alpha}\left(D_{x_{i}}^{2} u\right)^{2} \mathrm{~d} x\right)^{1+(\alpha / 2)} . \\
\cdot\left[(\operatorname{meas} \Omega)^{(2-\varrho) / 2 \beta}+\left(\int_{\Omega}\left(\left|D_{x_{i}} u\right|\right)^{4 \beta /(2-\varrho)} \mathrm{d} x\right)^{(2-\varrho) / 2 \beta}\right]^{\beta} \leqq \\
\leqq 2^{\beta}\left(\int_{\Omega}\left(1+\left(D_{x_{i}} u\right)^{2}\right)^{\alpha}\left(D_{x_{i}}^{2} u\right)^{2} \mathrm{~d} x\right)^{1+(\alpha / 2)} \cdot\left[(\text { meas } \Omega)^{|\alpha| / 2}+\left|D_{x_{i}} u\right|_{\Omega, 4+2 \alpha}^{(2+\alpha)|\alpha|}\right] .
\end{gathered}
$$

From (2.9) and (2.7)' the assertion follows immeadiately. q.e.d.

\section{AN A PRIORI ESTIMATE FOR REGULAR FUNCTIONS}

Let $\Omega$ be the set $] 0,1\left[{ }^{n} \subset R^{n}\right.$ and $\alpha$ a real number such that: $-\frac{1}{6} \leqq \alpha<0$.

We consider the following spaces of real functions:

$$
\begin{aligned}
& \mathscr{A}=\left\{a(x) \text { real valued: } a \in C^{1}(\bar{\Omega})\right\} \\
& \mathscr{U}=\left\{u(x) \text { real valued: } u \in C^{3}(\bar{\Omega}),\left.u\right|_{\partial \Omega}=0\right\} .
\end{aligned}
$$

Let $a_{1}(x), \ldots, a_{n}(x)$ be functions such that:

$$
a_{i} \in \mathscr{A}, \quad a_{i}(x) \geqq 1 \quad \forall x \in \Omega \quad(i=1, \ldots, n)
$$

and set:

$$
A=\sum_{i, k=1}^{n}\left|D_{x_{k}} a_{i}\right|_{\Omega, 4+2 \alpha} \equiv \sum_{i, k}\left[\int_{\Omega}\left|D_{x_{k}} a_{i}\right|^{4+2 \alpha} \mathrm{d} x\right]^{1 /(4+2 \alpha)}
$$

We introduce the elliptic operator: $\mathscr{L}=-\sum_{i=1}^{n} a_{i}(x) D_{x_{i}}^{2}$, which, in this section, will be regarded only as operator $\mathscr{L}: \mathscr{U} \rightarrow \mathscr{A}$.

Since $\Omega$ lies between the hyperplanes $\left\{x_{1}=0\right\}$ and $\left\{x_{1}=1\right\}$ and the ellipticity constant of $\mathscr{L}$ is $\lambda=1$, the classical maximum principle (see $\mathrm{G}$. T. Theorem 3.7 page $35[5])^{\prime}$ allows us to say that:

$$
\sup _{\Omega}|u| \leqq(\mathrm{e}-1) \sup _{\Omega}|\mathscr{L} u|, \quad \forall u \in \mathscr{U} .
$$

The aim of this section is to establish some estimates of the second derivatives of $u \in \mathscr{U}$ which involve, in the right-hand side, only $A$ and $\sup _{\Omega}|\mathscr{L} u|$. 
In spite of the rather unusual form of these estimates, the technique employed is that, by now classical, of the paper [8] of C. Miranda.

Nevertheless we feel that it is better to expose the proofs in details because of certain situations which are caused by the parameter $\alpha$ that here is negative, while in [8] is positive. First of all we introduce some notations, by putting [all the $u$ are real]:

$$
\forall u \in C^{1}(\bar{\Omega}), \quad U_{i}(x)=\left[1+\left(D_{x_{i}} u(x)\right)^{2}\right], \quad i=1, \ldots, n .
$$

It is evident that:

$$
\forall u \in C^{1}(\bar{\Omega}), \quad\left|D_{x_{i}} u\right| \leqq U_{i}^{1 / 2}
$$

We also define:

$$
\forall u \in C^{2}(\bar{\Omega}), \quad\|u\| \|=\left[\sum_{i, k=1}^{n} \int_{\Omega} U_{k}^{\alpha}\left(D_{x_{i}} D_{x_{k}} u\right)^{2} \mathrm{~d} x\right]^{1 / 2}
$$

where $\alpha \in[-1 / 6,0[$.

We remark that in the right-hand side of (3.5) there are not addenda like $U_{k}^{\alpha}\left(D_{x_{i}}^{2} u\right)^{2}$ with $i \neq k$.

We observe that \|\|$u \| \mid$ substitutes the integral $\boldsymbol{P}_{\alpha}$ in the proof of C. Miranda [8], whereas the function $\theta_{\alpha}$ of that proof ,,breaks" into the functions $U_{k}^{\alpha}$.

It is evident that the first factor of the first addendum in the right-hand side of $(2.7)^{\prime}$ is estimated by:

$$
\begin{gathered}
\left|\left(1+\left(D_{x_{i}} u\right)^{2}\right)^{\alpha / 2} D_{x_{i}}^{2} u\right|_{\Omega, 2} \equiv\left(\int_{\Omega} U_{i}^{\alpha}\left(D_{x_{i}}^{2} u\right)^{2} \mathrm{~d} x\right)^{1 / 2} \leqq\|u \mid\|, \\
\forall u \in C^{2}(\bar{\Omega}) \quad(i=1, \ldots, n) .
\end{gathered}
$$

It is obvious that, in particular, (3.3), (3.4), (3.5) and (3.6) hold for $u \in \mathscr{U}$. For any $u \in \mathscr{U}$, we define: $f=\mathscr{L} u \equiv-\sum_{i} a_{i} D_{x_{i}}^{2} u$. Then, multiplying both sides by $-U_{k}^{\alpha} D_{x_{k}}^{2} u$ and taking the integral over $\Omega$, we obtain:

$$
\int_{\Omega} a_{k} U_{k}^{\alpha}\left(D_{x_{k}}^{2} u\right)^{2} \mathrm{~d} x+\sum_{i \neq k}^{1 \leqq i \leqq n} \int_{\Omega} a_{i} U_{k}^{\alpha} D_{x_{i}}^{2} u D_{x_{k}}^{2} u \mathrm{~d} x=-\int_{\Omega} f U_{k}^{\alpha} D_{x_{k}}^{2} u \mathrm{~d} x .
$$

Integrating by parts, in virtue of the conditions: $u \in \mathscr{U}, a_{i} \in \mathscr{A}$ (see (3.1)), we easily have:

$$
\begin{gathered}
\int_{\Omega} a_{i} U_{k}^{\alpha} D_{x_{i}}^{2} u D_{x_{k}}^{2} u \mathrm{~d} x=-\int_{\Omega} D_{x_{k}} a_{i} U_{k}^{\alpha} D_{x_{i}}^{2} u D_{x_{k}} u \mathrm{~d} x- \\
-2 \alpha \int_{\Omega} a_{i} U_{k}^{\alpha-1}\left(D_{x_{k}} u D_{x_{k}}^{2} u\right) D_{x_{i}}^{2} u D_{x_{k}} u \mathrm{~d} x+ \\
+\int_{\Omega} a_{i} U_{k}^{\alpha}\left(D_{x_{i}} D_{x_{k}} u\right)^{2} \mathrm{~d} x+\int_{\Omega} D_{x_{i}} a_{i} U_{k}^{\alpha} D_{x_{i}} D_{x_{k}} u D_{x_{k}} u \mathrm{~d} x+ \\
+2 \alpha \int_{\Omega} a_{i} U_{k}^{\alpha-1}\left(D_{x_{k}} u D_{x_{i}} D_{x_{k}} u\right)^{2} \mathrm{~d} x .
\end{gathered}
$$

After this, by (3.7) (being: $-\sum_{i \neq k} a_{i} D_{x_{i}}^{2} u=a_{k} D_{x_{k}}^{2} u+f$ ) it follows:

$$
\begin{gathered}
\sum_{i=1}^{n} \int_{\Omega} a_{i} U_{k}^{\alpha}\left(D_{x_{i}} D_{x_{k}} u\right)^{2} \mathrm{~d} x=-\int_{\Omega} f U_{k}^{\alpha} D_{x_{k}}^{2} u \mathrm{~d} x+ \\
+\sum_{i \neq k}^{1 \leqq i \leqq n} \int_{\Omega} D_{x_{k}} a_{i} U_{k}^{\alpha} D_{x_{k}} u D_{x_{i}}^{2} u \mathrm{~d} x-\sum_{i \neq k}^{1 \leqq i \leqq n} \int_{\Omega} D_{x_{i}} a_{i} U_{k}^{\alpha} D_{x_{k}} u D_{x_{i}} D_{x_{k}} u \mathrm{~d} x-
\end{gathered}
$$




$$
\begin{gathered}
-2 \alpha \sum_{i \neq k}^{1 \leqq i \leqq n} \int_{\Omega} a_{i} U_{k}^{\alpha-1}\left(D_{x_{k}} u\right)^{2}\left(D_{x_{i}} D_{x_{k}} u\right)^{2} \mathrm{~d} x- \\
-2 \alpha \int_{\Omega} U_{k}^{\alpha-1}\left(D_{x_{k}} u\right)^{2} a_{k}\left(D_{x_{k}}^{2} u\right)^{2} \mathrm{~d} x-2 \alpha \int_{\Omega} U_{k}^{\alpha-1}\left(D_{x_{k}} u\right)^{2} D_{x_{k}}^{2} u \cdot f \mathrm{~d} x .
\end{gathered}
$$

From here, remembering (3.3) and (3.4), and taking the sum over $k=1, \ldots, n$, we get:

$$
\begin{gathered}
\sum_{i, k=1}^{n} \int_{\Omega} a_{i} U_{k}^{\alpha}\left(D_{x_{i}} D_{x_{k}} u\right)^{2} \mathrm{~d} x \leqq \int_{\Omega} \sum_{k=1}^{n}\left|f U_{k}^{\alpha} D_{x_{k}}^{2} u\right| \mathrm{d} x+ \\
+\sum_{i, k \leqq n}^{i \neq k} \int_{\Omega}\left|D_{x_{k}} a_{i} D_{x_{k}} u U_{k}^{\alpha} D_{x_{i}}^{2} u\right| \mathrm{d} x+ \\
+\sum_{i, k \leqq n}^{i \neq k} \int_{\Omega}\left|D_{x_{i}} a_{i} D_{x_{k}} u U_{k}^{\alpha} D_{x_{i}} D_{x_{k}} u\right| \mathrm{d} x+ \\
+2|\alpha|\left\{\sum_{i, k \leqq n}^{i \neq k} \int_{\Omega} a_{i} U_{k}^{\alpha}\left(D_{x_{i}} D_{x_{k}} u\right)^{2} \mathrm{~d} x+\sum_{k=1}^{n} \int_{\Omega} a_{k} U_{k}^{\alpha}\left(D_{x_{k}}^{2} u\right)^{2} \mathrm{~d} x\right\}+ \\
+2|\alpha| \sum_{k=1}^{n} \int_{\Omega} U_{k}^{\alpha}\left[\frac{1}{2}\left(D_{x_{k}}^{2} u\right)^{2}+\frac{1}{2} f^{2}\right] \mathrm{d} x .
\end{gathered}
$$

Moreover, since $\stackrel{a}{a}_{k} \geqq 1$ (see (3.1)) we have:

and hence:

$$
\frac{1}{2}\left(D_{x_{k}}^{2} u\right)^{2} \leqq \frac{1}{2} \sum_{i=1}^{n} a_{i}\left(D_{x_{i}} D_{x_{k}} u\right)^{2},
$$

$$
\begin{gathered}
\sum_{i, k=1}^{n} \int_{\Omega} a_{i} U_{k}^{\alpha}\left(D_{x_{i}} D_{x_{k}} u\right)^{2} \mathrm{~d} x \leqq \int_{\Omega} \sum_{k=1}^{n}\left|f U_{k}^{\alpha} D_{x_{k}}^{2} u\right| \mathrm{d} x+ \\
+\sum_{i, k \leqq n}^{i \neq k} \int_{\Omega}\left|D_{x_{k}} a_{i} D_{x_{k}} u U_{k}^{\alpha} D_{x_{i}}^{2} u\right| \mathrm{d} x+\sum_{i, k \leqq n}^{i \neq k} \int_{\Omega}\left|D_{x_{i}} a_{i} D_{x_{k}} u U_{k}^{\alpha} D_{x_{i}} D_{x_{k}} u\right| \mathrm{d} x+ \\
+3|\alpha| \sum_{i, k=1}^{n} \int_{\Omega} a_{i} U_{k}^{\alpha}\left(D_{x_{i}} D_{x_{k}} u\right)^{2} \mathrm{~d} x+|\alpha| \sum_{k} \int_{\Omega} U_{k}^{\alpha} f^{2} \mathrm{~d} x .
\end{gathered}
$$

On the basis of (3.9) and (3.2) we prove the following:

Theorem 3.I. Let $\mathscr{C} \equiv-\sum_{i=1}^{n} a_{i} D_{x_{i}}^{2}$ be an elliptic operator satisfying (3.1). If: $-1 / 6 \leqq \alpha<0$, then there exists a constant $c(n)$ depending only on $n$ such that, for every $i=1, \ldots, n$ :

$$
\begin{gathered}
\int_{\Omega}\left|D_{x_{i}}^{2} u\right|^{2+\alpha} \mathrm{d} x \leqq c(n) H(\Psi, \Phi) \equiv \\
\equiv c(n)\left\{\left[\Psi^{2}+\Phi^{2}+\Psi^{4+2 \alpha} \Phi^{2+2 \alpha}\right]^{1+(\alpha / 2)}+\right. \\
\left.+\Phi^{|\alpha|}\left[\Psi^{2}+\Phi^{2}+\Psi^{4+2 \alpha} \Phi^{2+2 \alpha}\right]\right\},
\end{gathered}
$$

$\forall u \in \mathscr{U}$, whatever the numbers: $\Psi \geqq A, \Phi \geqq \sup _{\Omega}|\mathscr{L} u| \equiv \sup _{\Omega}|f|$ are.

Proof. First of all we fix $\eta=1 / 4$ so that the numbers $p=2, \eta=1 / 4, \alpha \in[-1 / 6,0[$ satisfy $(1.1)_{\infty}$. Consequently we can use $(2.7)^{\prime}$ of Corollary 2 .III written for $(p, \eta, \alpha) \equiv$ $\equiv\left(2, \frac{1}{4}, \alpha\right)$. In particular $\bar{K}_{3}=\bar{K}_{3}(1 / 4)=2^{75+2 / 5}$ is now an absolute constant. All this understood we start the proof. Since $\alpha<0$, we have: $\left[1+\left(D_{x_{j}} u\right)^{2}\right]^{\alpha} \equiv$ 
$\equiv U_{j}^{\alpha} \leqq U_{j}^{\alpha / 2} \leqq 1$. Moreover, being $|\alpha| \leqq 1 / 6$ and $a_{i} \geqq 1$, from (3.9) we get:

$$
\begin{aligned}
\|\left.|u|\right|^{2} \leqq & 2 \sum_{k=1}^{n} \int_{\Omega}|f|\left|U_{k}\right|^{\alpha / 2}\left|D_{x_{k}}^{2} u\right| \mathrm{d} x+\frac{n}{3}\left(\sup _{\bar{\Omega}}|f|\right)^{2}+ \\
& +2 \sum_{i, k \leqq n}^{i \neq k} \int_{\Omega}\left|D_{x_{k}} a_{i} D_{x_{k}} u U_{k}^{\alpha} D_{x_{i}}^{2} u\right| \mathrm{d} x+ \\
& +2 \sum_{i, k \leqq n}^{i \neq k} \int_{\Omega}\left|D_{x_{i}} a_{i} D_{x_{k}} u U_{k}^{\alpha} D_{x_{i}} D_{x_{k}} u\right| \mathrm{d} x .
\end{aligned}
$$

Applying Hölder's inequality with the exponents $4+2 \alpha,(4+2 \alpha) /(1+\alpha)$, 2, we obtain:

$$
\begin{gathered}
\int_{\Omega}\left|D_{x_{i}} a_{i} D_{x_{k}} u U_{k}^{\alpha} D_{x_{i}} D_{x_{k}} u\right| \mathrm{d} x \leqq \\
\leqq \int_{\Omega}\left|D_{x_{i}} a_{i}\right|\left|D_{x_{k}} u\right|^{1+\alpha}\left|U_{k}^{\alpha / 2} D_{x_{i}} D_{x_{k}} u\right| \mathrm{d} x \leqq\left(\int_{\Omega}\left|D_{x_{i}} a_{i_{i}}\right|^{4+2 \alpha} \mathrm{d} x\right)^{1 /(4+2 \alpha)} . \\
\cdot\left[\left(\int_{\Omega}\left|D_{x_{k}} u\right|^{4+2 \alpha} \mathrm{d} x\right)^{1 /(4+2 \alpha)}\right]^{1+\alpha} \cdot\left(\int_{\Omega} U_{k}^{\alpha}\left(D_{x_{i}} D_{x_{k}} u\right)^{2} \mathrm{~d} x\right)^{1 / 2} \leqq \\
\leqq\left|D_{x_{i}} a_{i}\right|_{\Omega, 4+2 \alpha}\left|D_{x_{k}} u\right|_{2,4+2 \alpha}^{1+\alpha}|| u|| \mid .
\end{gathered}
$$

In estimating the third addendum in the right-hand side of (3.11) a question immediately arises: here there are the terms $U_{k}^{\alpha / 2} D_{x_{i}}^{2} u(i \neq k)$ whose squares (as already noticed) do not appear in the expression of \|\|$u \| \mid(\operatorname{see}(3.5))$. But the problem is solved multiplying and dividing by a suitable factor and applying Hölder's inequality with the exponents:

$$
\begin{gathered}
s=\frac{4+2 \alpha}{1+2 \alpha}, \quad \sigma=\frac{4+2 \alpha}{|\alpha|}, \quad r=4+2 \alpha, \quad 2: \\
\int_{\Omega}\left|D_{x_{k}} a_{i} D_{x_{k}} u U_{k}^{\alpha} D_{x_{i}}^{2} u\right| \mathrm{d} x= \\
=\int_{\Omega}\left|D_{x_{k}} a_{i} D_{x_{k}} u U_{k}^{\alpha}\left[1+\left(D_{x_{i}} u\right)^{2}\right]^{|\alpha| / 2} U_{i}^{\alpha / 2} D_{x_{i}}^{2} u\right| \mathrm{d} x \leqq \\
\leqq\left(\int_{\Omega}\left|D_{x_{k}} a_{i}\right|^{r} \mathrm{~d} x\right)^{1 / r}\left(\int_{\Omega} \frac{\left|D_{x_{k}} u\right|^{s} \mathrm{~d} x}{\left.\left[1+\left(D_{x_{k}} u\right)^{2}\right]^{|\alpha| s}\right)^{1 / s} .}\right. \\
.\left(\int_{\Omega}\left[1+\left(D_{x_{i}} u\right)^{2}\right]^{|\alpha| \sigma / 2} \mathrm{~d} x\right)^{1 / \sigma}\left(\int_{\Omega} U_{i}^{\alpha}\left(D_{x_{i}}^{2} u\right)^{2} \mathrm{~d} x\right)^{1 / 2} \leqq \\
\leqq\left(\int_{\Omega}\left|D_{x_{k}} a_{i}\right|^{r} \mathrm{~d} x\right)^{1 / r}\left[\left(\int_{\Omega}\left|D_{x_{k}} u\right|^{(1+2 \alpha) s} \mathrm{~d} x\right)^{1 /(1+2 \alpha) s}\right]^{1+2 \alpha} . \\
.\left[2^{|\alpha| \sigma / 2} \int_{\Omega_{i, u^{1}}}\left|D_{x_{i}} u\right|^{|\alpha| \sigma} \mathrm{d} x+2^{|\alpha| \sigma / 2} \operatorname{meas}\left\{x \in \Omega:\left|D_{x_{i}} u\right| \leqq 1\right\}\right]^{1 / \sigma} . \\
.\left(\int_{\Omega} U_{i}^{\alpha}\left(D_{x_{i}}^{2} u\right)^{2} \mathrm{~d} x\right)^{1 / 2} \leqq 2^{|\alpha|}\left|D_{x_{k}} a_{i}\right|_{\Omega, 4+2 \alpha}\left|D_{x_{k}} u\right|_{\Omega, 4+2 \alpha}^{1+2 \alpha}\left\{\left|D_{x_{i}} u\right|_{\Omega, 4+2 \alpha}^{|\alpha|}+1\right\}|||u||| .
\end{gathered}
$$

To obtain (3.13) we have also used the second inequality of (1.2) and the fact that:

$$
\alpha \geqq-\frac{1}{6} \Rightarrow \sigma=\frac{4+2 \alpha}{|\alpha|}>\frac{2}{|\alpha|} \Rightarrow \frac{1}{\sigma}+\frac{|\alpha|}{2}<|\alpha| .
$$

From $(2.7)^{\prime}$, Corollary 2.III (where $\bar{K}_{3}=2^{75+2 ! 5}$ ), (3.5), (3.6), (3.2), (3.12), (3.13) we get:

$$
\begin{aligned}
& \int_{\Omega}\left|D_{x_{i}} a_{i} D_{x_{i}} u U_{k}^{\alpha} D_{x_{i}} D_{x_{k}} u\right| \mathrm{d} x \leqq \bar{K}_{3}\left|D_{x_{i}} a_{i}\right|_{\Omega, 4+2 \alpha} . \\
& \cdot\left[|| u \|\left.\right|^{1 /(\alpha+2)}\left(2 \sup _{\Omega}|f|\right)^{1 /(\alpha+2)}+1\right]^{1+\alpha}|| u \mid \|,
\end{aligned}
$$




$$
\begin{gathered}
\int_{\Omega}\left|D_{x_{k}} a_{i} D_{x_{k}} u U_{k}^{\alpha} D_{x_{i}}^{2} u\right| \mathrm{d} x \leqq 2^{1+|\alpha|} \bar{K}_{3}\left|D_{x_{k}} a_{i}\right|_{\Omega, 4+2 \alpha} . \\
.\left[|||u \||||^{1 /(\alpha+2)}\left(2 \sup _{\Omega}|f|\right)^{1 /(\alpha+2)}+1\right]^{1+\alpha} \mid\|u\| \|,
\end{gathered}
$$

where: $f=\mathscr{L} u \equiv-\sum_{i=1}^{n} a_{i} D_{x_{i}}^{2} u$.

From (3.11), (3.12)' and (3.13)' it follows easily [with the constant $A$ of (3.1)']:

$$
\begin{aligned}
\left.\|u\|\right|^{2} \leqq & (n / 3)\left(\sup _{\Omega}|f|\right)^{2}+2 n\left(\sup _{\Omega} f\right)\|u\| \|+\left(2 n+2^{2+\alpha}\right) \bar{K}_{3} 2^{1+\alpha} A . \\
& \cdot\left[\|\| u \|\left.\right|^{(1+\alpha) /(2+\alpha)}\left(2 \sup _{\Omega}|f|\right)^{(1+\alpha) /(2+\alpha)}+1\right]\|u\| \| ;
\end{aligned}
$$

writing, for sake of brevity $\xi=\|\| u \|\left.\right|^{2}$ and putting:

$$
K(n)=(4 n+8) 2^{75+2 / 5}\left[\geqq\left(2 n+2^{2+|\alpha|}\right) 2^{1+\alpha} \bar{K}_{3}\right],
$$

from (3.14) we have:

$$
\begin{aligned}
\xi \leqq & n / 3\left(\sup _{\Omega}|f|\right)^{2}+\left[2 n\left(\sup _{\Omega}|f|\right)+K(n) A\right] \xi^{1 / 2}+ \\
= & +2 K(n) A\left(\sup _{\Omega}|f|\right)^{(1+\alpha) /(2+\alpha)} \xi^{(3+2 \alpha) /(4+2 \alpha)} .
\end{aligned}
$$

From $(3.14)^{\prime}$, applying the first inequality of (1.2), with $q_{1}=q_{2}=q_{3}^{\prime}=3$, we deduce:

$$
\begin{gathered}
\xi \equiv \sum_{i, k=1}^{n} \int_{\Omega} U_{k}^{\alpha}\left(D_{x_{i}} D_{x_{k}} u\right)^{2} \mathrm{~d} x \leqq \\
\leqq n\left(\sup _{?}|f|\right)^{2}+9\left[2 n\left(\sup _{\Omega}|f|\right)+K(n) A\right]^{2}+ \\
\left.+[6 K(n) A]^{4+2 \alpha} \sup _{\Omega}|f|\right)^{2+2 \alpha} \leqq \\
\leqq K_{1}(n)\left[\left(\sup _{\Omega}|f|\right)^{2}+A^{2}+A^{4+2 \alpha}\left(\sup _{\Omega}|f|\right)^{2+2 \alpha}\right],
\end{gathered}
$$

where: $K_{1}(n)=\left(n+72 n^{2}\right)+18 K(n)^{2}+1296 K(n)^{4}$. Finally by $(2.8)$ of theorem 2.II, (3.2), (3.6) and (3.15) we get [using that: $K_{1}(n)>1, \bar{K}_{3}>1,-1 / 6 \leqq \alpha<0$ ]:

$$
\begin{gathered}
\int_{\Omega}\left|D_{x_{i}}^{2} u\right|^{2+\alpha} \mathrm{d} x \leqq 2 K_{1}(n) . \\
\cdot\left[\left(\sup _{\Omega}|f|\right)^{2}+A^{2}+A^{4+2 \alpha}\left(\sup _{\Omega}|f|\right)^{2+2 \alpha}\right]^{1+\alpha / 2} . \\
\cdot\left\{1+\bar{K}_{3}\left[1+\left(2 \sup _{\Omega}|f|\right)^{1 /(\alpha+2)} K_{1}(n) .\right.\right. \\
\left.\left..\left(\left(\sup _{\Omega}|f|\right)^{2}+A^{2}+A^{4+2 \alpha}\left(\sup _{\Omega}|f|\right)^{2+2 \alpha}\right)^{1 /(4+2 \alpha)}\right]^{(2+\alpha)|\alpha|}\right\} \leqq \\
8 \bar{K}_{3} K_{1}(n)^{2} H\left(A, \sup _{\Omega}|f|\right) \equiv \\
\equiv c(n) H\left(A, \sup _{\Omega}|f|\right) \equiv c(n) H\left(A, \sup _{\Omega}|\mathscr{L} u|\right) .
\end{gathered}
$$

From $(3.10)^{\prime}$ the assertion follows once we observe that the function $H(\Psi, \Phi)$ of (3.10) is increasing in $\Psi$, for every fixed $\Phi$, and increasin in $\Phi$ for every fixed $\Psi$. q.e.d.

Using Theorem 3.I we estimate the norms $L^{2+\alpha}$ of the pure second derivatives of 
$u \in \mathscr{U}$, by means of the $\sup _{\Omega} \mathscr{L}|u|$ and $A=\sum_{i, k}\left|D_{x_{k}} a_{i}\right|_{\Omega, 4+2 \alpha}$. To estimate the mixed second derivatives of $u$, as well as the first derivatives, we proceed in the usual way.

Actually the following theorem holds:

Theorem 3.II. Under the same hypotheses of Theorem 3.I we have:

$$
\begin{gathered}
\left|D^{2} u\right|_{\Omega, 2+\alpha}+\left|D^{1} u\right|_{\Omega, 4+2 \alpha}+\sup _{\Omega}|u| \leqq \\
\leqq c_{1}(n, \alpha)\left[\{c(n) H(\Psi, \Phi)\}^{1 /(2+\alpha)}+\Phi\right], \forall u \in \mathscr{U},
\end{gathered}
$$

whatever the numbers $\Psi \geqq A \equiv \sum_{i, k}\left|D_{x_{k}} a_{i}\right|_{\Omega, 4+2 \alpha}, \Phi \geqq \sup _{\Omega}|\mathscr{L} u|$ are and with $c_{1}(n, \alpha)$ a constant which depends only on $n$ and $\alpha$.

Proof. To estimate the seminorm $\left|D^{2} u\right|_{\Omega, 2+\alpha}$, we can, for example, utilize Theorem 4.3 (second part) of M. Troisi [15] (,,Teoremi di inclusione per Spazi di Sobolev non isotropi" - Ric. Mat. vol. XVIII - 1969) in the case: $\left.r=n ; \Omega_{i}=\right] 0,1[$ for $i=1, \ldots, r ; m_{1}=m_{2}=\ldots=m_{n}=2 ; p_{1}=\ldots=p_{n}=2+\alpha$.

Then in the formula (4.7) of [15] [and in (2.2)] we have: $s=2+\alpha$ in the lefthand side, while the right-hand side (see (4.3) and (4.4)) is estimated by:

$$
\sum_{i=1}^{n}\left|D_{x_{i}}^{2} u\right|_{\Omega, 2+\alpha}+2 n \sup _{\Omega}|\mathscr{L} u|
$$

in virtue of (3.2) of the present paper.

Thus (3.10) of the previous Theorem gives the required estimate for $\left|D^{2} u\right|_{\Omega, 2+\alpha}$. Finally, to estimate the first derivatives (that is $\left|D^{1} u\right|_{\Omega, 4+2 \alpha}$ ) it is enough to apply Gagliardo-Nirenberg's inequality [11]. q.e.d.

\section{AN EXISTENCE THEOREM}

The estimate (3.16) has been obtained for functions $u \in C^{3}(\bar{\Omega})$ which vanish on $\partial \Omega$ and for an operator $\mathscr{L} \equiv-\sum_{i} a_{i} D_{x_{i}}^{2}$ with coefficients belonging to $C^{1}(\bar{\Omega})$. Nevertheless in (3.16) only the norms $L^{2+\alpha}$ of the second derivatives of $u$ and the $L^{4+2 \alpha}$ norms of the first derivatives of the $a_{i}$ 's (as well as the $L^{\infty}$ norm of $\mathscr{L} u$ ) appear.

This will allow us to establish an existence Theorem for the equation $\mathscr{L} u=f$ in $\Omega \equiv] 0,1\left[{ }^{n}\right.$, under the only hypothesis:

$$
a_{i} \in W^{1,4+2 \alpha}(\Omega) \text { with: }-1 / 6 \leqq \alpha<0 ;
$$

$a_{i}(x)$ real $\geqq \lambda>0, \forall x \in \Omega, i=1, \ldots, n, n \geqq 4$ [for $n=3$ it is enough to apply Shauder's theory]. ${ }^{1}$ ) To this aim it will be necessary to use some existence and uniqueness theorems with much stronger assumptions on the coefficients. Let us start by introducing some classes of functions. We denote by $v$ the outer normal to

1) Remark that (4.1) does not require the coefficients to be bounded. 
$\partial \Omega$ (in the regular points of $\partial \Omega$ ) and set:

$$
\begin{gathered}
C_{0}^{\text {even }}(\Omega)=\left\{u \text { real: } u \in C^{\infty}(\bar{\Omega}),\left.D_{v}^{2 r} u\right|_{\partial \Omega}=0 \forall r \in N\right\}, \\
\Omega=] 0,1\left[{ }^{n}, \text { where : } N=\{0,1,2, \ldots\} \quad\left(\text { while } N_{0}=\{1,2, \ldots\}\right) .\right.
\end{gathered}
$$

Clearly we have:

$$
\begin{gathered}
\forall k \equiv\left(k_{1}, \ldots, k_{n}\right) \in N_{0}^{n}, \\
u_{k}(x) \equiv \prod_{i=1}^{n} u_{k_{i}}\left(x_{i}\right) \equiv \prod_{i=1}^{n} \operatorname{sen} K_{i} \pi x_{i} \in C_{0}^{\text {even }}(\Omega) .
\end{gathered}
$$

Let $\mu \equiv\left(\mu_{1}, \ldots, \mu_{n}\right)$ be $n$ real numbers greater then 1 and $L_{\mu}$ the elliptic operator with constant coefficients:

It is obvious that:

$$
L_{\mu}=-\sum_{i=1}^{n} \mu_{i} D_{x_{i}}^{2}
$$

$$
\forall u \in C_{0}^{\mathrm{even}}(\Omega), \quad L_{\mu} u \in C_{0}^{\mathrm{even}}(\Omega) .
$$

On the other hand, using the classical method of Fourier, we have:

$$
\forall f \in C_{0}^{\text {even }}(\Omega), \quad \exists \mid u \in C_{0}^{\text {even }}(\Omega): L_{\mu} u=f
$$

(obviously $\left.u\right|_{\partial \Omega}=0$ ) [from now on the symbol $\exists \mid$ means "there exists a unique"]. Such a $u$ (unique) is given by (see (4.2)):

$$
u(x)=\sum_{k \in N_{0}{ }^{n}}\left[\pi^{-2}\left(\sum_{i=1}^{n} \mu_{i} K_{i}^{2}\right)^{-1} \int_{\Omega} 2^{n / 2} f(\xi) u_{k}(\xi) \mathrm{d} \xi\right] 2^{n / 2} u_{k}(x)
$$

$\left[\sum_{k \in N_{0}{ }^{n}}\right.$ means: $\lim _{m \rightarrow \infty} \sum_{k \in A_{m}}\left(\lim\right.$ in $\left.L^{2}(\Omega)\right)$ where $A_{m}=\psi^{-1}(\{1, \ldots, m\})$, and $\psi: N_{0}^{n} \rightarrow N_{0}$ is a bijection]. Hence, for each $r \in N$, we have:

$$
\begin{gathered}
\forall u \in C_{0}^{\mathrm{even}}(\Omega), \\
\|u\|_{W^{2 r+2,2}(\Omega)} \leqq c_{n, r}\left\|L_{\mu} u\right\|_{W^{2 r, 2}(\Omega)}
\end{gathered}
$$

where $C_{n, r}$ is a constant which depends only $n$ and $r$. For each $r \in N$ we denote by $\mathscr{P}^{2 r, 2}(\Omega)$ the closure of $C_{0}^{\text {even }}(\Omega)$ in the Sobolev space $W^{2 r, 2}(\Omega)$. Since $C_{0}^{\text {even }}(\Omega)$ is dense in $\mathscr{P}^{2 r, 2}(\Omega)$, on the basis of (4.4) and (4.4)" we prove the following:

Proposition 4.I. Let $\mu_{1}, \ldots, \mu_{n}$ be real numbers $\geqq 1$ and $m=2 r>1+n / 2$ $(r \in N)$. Then we have:

$$
\begin{gathered}
\forall f \in \mathscr{P}^{m, 2}(\Omega), \quad \exists \mid u \in \mathscr{P}^{m+2,2}(\Omega): \\
L_{\mu} u=-\sum_{i=1}^{n} \mu_{i} D_{x_{i}}^{2} u=f .
\end{gathered}
$$

Moreover:

$$
\begin{gathered}
\forall u \in \mathscr{P}^{m+2,2}(\Omega), \\
\|u\|_{W^{m+2,2}(\Omega)} \leqq c_{n, r}\left\|L_{\mu} u\right\|_{W^{m, 2}(\Omega)}
\end{gathered}
$$


Finally (obviously):

$$
\begin{gathered}
\forall u \in \mathscr{P}^{m+2,2}(\Omega), \\
u \in C^{3}(\bar{\Omega}) \text { and }\left.u\right|_{\partial \Omega}=0 .
\end{gathered}
$$

Now we consider an elliptic operator $\mathscr{L}_{1}=-\sum_{i=1}^{n} a_{i}(x) D_{x_{i}}^{2}$ satisfying the conditions:

$$
\begin{aligned}
& a_{i} \in C^{\infty}(\bar{\Omega}) ; \quad a_{i}(x) \geqq 1 \quad \forall x \in \Omega ; \\
& \exists \eta \in] 0, \frac{1}{2}\left[: \forall\left(x_{1}, \ldots, x_{n}\right) \in \bar{\Omega}, \forall j \in\{1, \ldots, n\},\right. \\
& \left.x_{j} \in[0, \eta] \cup[1-\eta, 1] \Rightarrow D_{x_{j}} a_{i}\left(x_{1}, \ldots, x_{n}\right)=0\right) ;
\end{aligned}
$$

which, in particular, imply that $a_{i}(x)$ is constant in the set $[0, \eta]^{n}$. We also consider, for $\sigma \in[0,1]$ the operator:

$$
\mathscr{L}_{\sigma}=\sum_{i=1}^{n} a_{i}^{\sigma}(x) D_{x_{i}}^{2} \equiv-\sum_{i=1}^{n}\left[\sigma a_{i}(x)+1-\sigma\right] D_{x_{i}}^{2}
$$

whose coefficients satisfy (like the $a_{i}$ 's) (4.6). We easily see (from (4.6)) that:

$$
\forall u \in C_{0}^{\text {even }}(\Omega), \quad \mathscr{L}_{\sigma} u \in C_{0}^{\text {even }}(\Omega) .
$$

From (4.7) it follows, for each $r \in N$ :

$$
\forall \sigma \in[0,1], \quad \mathscr{L}_{\sigma} \in \mathscr{L}\left(\mathscr{P}^{2 r+2,2}(\Omega), \mathscr{P}^{2 r, 2}(\Omega)\right) .
$$

Moreover, if $m=2 r>1+n / 2$, using $(4.5)^{\prime}$, the classical ,Korn's device” and the Maximum principle (see, for example, G.T. [5]) we prove that:

$$
\begin{gathered}
\forall u \in \mathscr{P}^{m+2,2}(\Omega), \quad \forall \sigma \in[0,1], \\
\|u\|_{W^{m+2,2}(\Omega)} \leqq K\left\|\mathscr{P}_{\sigma} u\right\|_{W^{m, 2}(\Omega)}
\end{gathered}
$$

with $K$ idependent of $u$ and $\sigma$, but depending on $n, m$ and the norms $\left\|a_{i}\right\|_{c^{m}(\bar{\Omega})}$.

From (4.7)" and proposition 4.I, in virtue of the ,continuation method" we get the following:

Theorem 4.I. Let $\mathscr{L}_{1} \equiv-\sum_{i} a_{i} D_{x_{i}}^{2}$ be an operator satisfying (4.6) and $m=$ $=2 r>1+n / 2$. Then:

$$
\forall f \in \mathscr{P}^{m, 2}(\Omega), \quad \exists \mid u \in \mathscr{P}^{m+2,2}(\Omega): \mathscr{L}_{1} u=f ;
$$

obviously: $\left.u\right|_{\partial \Omega}=0 ; u \in C^{3}(\bar{\Omega})$.

Now we are able to prove the following: Theorem 4.II. Let $\Omega$ be $] 0,1\left[{ }^{n}(\right.$ with $n \geqq 4)$ and $\mathscr{L} \equiv-\sum_{i=1}^{n} a_{i}(x) D_{x_{i}}^{2}$ an operator
satisfying the conditions:

$$
\begin{aligned}
& a_{i} \in W^{1,4+2 \alpha}(\Omega), \quad-\frac{1}{6} \leqq \alpha<0 \\
& \exists \lambda>0: \forall x \in \Omega, \quad a_{i}(x) \text { real } \geqq \lambda,
\end{aligned}
$$


$i=1,2, \ldots, n$. Then we have:

$$
\begin{gathered}
\forall f \in L^{\infty}(\Omega), \quad \exists u \in W^{2,2+\alpha}(\Omega) \cap L^{\infty}(\Omega): \\
\mathscr{L} u=f \text { in } \Omega,\left.\quad u\right|_{\partial \Omega}=0 \\
\sup _{\Omega}|u| \leqq(\mathrm{e}-1) \sup _{\Omega}|f| .
\end{gathered}
$$

Proof. Without loss of generality, we suppose that: $\lambda=1$. Fixed arbitratily $\varepsilon>0$, we determine a function $f_{\varepsilon}$ such that:

$$
\begin{gathered}
f_{\varepsilon} \in C_{0}^{\infty}(\Omega) \subset C_{0}^{\text {even }}(\Omega) ; \\
\left\|f_{\varepsilon}-f\right\|_{L^{2}(\Omega)}<\varepsilon ; \quad \sup _{\Omega}\left|f_{\varepsilon}\right| \leqq \sup _{\Omega}|f| .
\end{gathered}
$$

Let us try to approximate the coefficients $a_{i}$ with some functions satisfying (4.6). Let $g_{i}$ be a function such that:

$$
\begin{gathered}
g_{i} \in C^{\infty}(\bar{\Omega}) ; \quad g_{i}(x) \geqq 1 \quad \forall x \in \Omega ; \\
\left\|a_{i}-g_{i}\right\|_{W^{1,4+2 \alpha}(\Omega)}<\varepsilon / 3 .
\end{gathered}
$$

We set, for each $\eta \in] 0,1 / 2[$ :

$$
\Omega^{\eta}=\{x \in \Omega: \operatorname{dist}(x, \partial \Omega) \geqq \eta\} \equiv[\eta, 1-\eta]^{n},
$$

and denote, for $x \in R^{n}$, by $x^{\eta} \in \Omega^{\eta}$ the point of minimum distance of $\Omega^{\eta}$ from $x$.

Then we introduce the function: $\tilde{g}_{i}(x)=g_{i}\left(x^{\eta}\right), x \in \mathbb{R}^{n}$. It is soon verified that: $\tilde{g}_{i} \in C^{0}\left(\mathbb{R}^{n}\right), \tilde{g}_{i}(x) \geqq 1 \forall x \in \mathbb{R}^{n}$. Moreover, for each straight line $r$ parallel to the $x_{j}$ axis $(j=1, \ldots, n), \tilde{g}_{i}$ is constant on both the half-lines

$$
\left\{\left(x_{1}, \ldots, x_{n}\right) \in r: x_{j} \leqq \eta\right\}, \quad\left\{\left(x_{1}, \ldots, x_{n}\right) \in r: x_{j} \geqq 1-\eta\right\} .
$$

Denoting by $\mathbb{R}_{j \eta}^{n}=\left\{x \in \mathbb{R}^{n}: x_{j} \neq \eta\right.$ and $\left.x_{j} \neq 1-\eta\right\}, g_{i}$ is also differentiable (in the classical meaning) with respect to $x_{j}$ in $\mathbb{R}_{j \eta}^{n}(j=1, \ldots, n)$. Moreover if, as in $[10],\left[D_{x_{j}} \psi\right]$ represents the classical derivative and $D_{x_{j}} \psi$ the derivative in the sense of distributions, we have:

Consequently:

$$
\begin{gathered}
{\left[D_{x_{j}} \tilde{g}_{i}\right](x)=0 \text { for } x_{j}<\eta \text { and for } x_{j}>1-\eta ;} \\
{\left[D_{x_{j}} \tilde{g}_{i}\right](x)=\left[D_{x_{j}} g_{i}\right]\left(x^{\eta}\right) \text { for } \eta<x_{j}<1-\eta .}
\end{gathered}
$$

$$
\sup _{\boldsymbol{R}_{j \eta^{n}}}\left|\left[D_{x_{j}} \tilde{g}_{i}\right]\right|=\max _{\Omega^{\boldsymbol{n}}}\left|\left[D_{x_{j}} g_{i}\right]\right|<+\infty,
$$

and hence (since $\tilde{g}_{i} \in C^{0}\left(\mathbb{R}^{n}\right)$ : see, for example, J. Nečas [10] Theorem 2.2 page 61):

$\forall p^{\prime}, \forall$ open bounded $\Omega^{\prime} \subset \mathbb{R}^{n}, \tilde{g}_{i} \in W^{1, p^{\prime}}\left(\Omega^{\prime}\right)$;

$D_{x_{j}} \tilde{g}_{i}=\left[D_{x_{j}} \tilde{g}_{i}\right]$ in $\Omega^{\prime}$ and even in the whole $\mathbb{R}^{n}(j=1, \ldots, n)$;

$$
\sup _{\boldsymbol{R}_{j \eta^{n}}}\left|D_{x_{j}} \tilde{a}_{i}\right| \leqq \max _{\bar{\Omega}}\left|\left[D_{x_{j}} g_{i}\right]\right|<+\infty \quad(j=1, \ldots, n) \text {. }
$$

Therefore, choosing $\eta \equiv \eta_{\varepsilon}$ small enough, we obtain:

$$
\left\|g_{i}-\tilde{g}_{i}\right\|_{W^{1,4+2 \alpha(\Omega)}} \equiv\left\|g_{i}-\tilde{g}_{i}\right\|_{W^{1,4+2 \alpha}\left(\Omega-\Omega^{\eta}\right)}<\varepsilon / 3 .
$$


After this (fixed $\tilde{g}_{i}[$ that is $\eta]$ in such a way that $(4.11)^{\prime}$ holds) we take $\Omega^{\prime} \supset \supset \Omega \equiv$ $\equiv] 0,1\left[{ }^{n}\right.$ and $p^{\prime}=4+2 \alpha$. Then if choose $\sigma \equiv \sigma_{\varepsilon}$ sufficiently small (see Lemma 3.15 Adams [1] page 52) we can manage to have, with a mollifier $\chi(x)$ :

$$
\begin{gathered}
\left\|a_{i}^{\varepsilon}-\tilde{g}_{i}\right\|_{W^{1,4+2 \alpha}(\Omega)}<\varepsilon / 3, \\
a_{i}^{\varepsilon}(x)=\sigma^{-n} \int_{\mathbb{R}^{n}} \chi\left(\frac{x-y}{\sigma}\right) \tilde{g}_{i}(y) \mathrm{d} y \in C^{\infty}\left(\mathbb{R}^{n}\right) .
\end{gathered}
$$

Finally if we take also $\sigma<\eta / 2$, we have that $a_{i}^{\varepsilon}$ is constant on both the half-lines $\left\{\left(x_{1}, \ldots, x_{n}\right) \in r: x_{j}<\eta / 2\right\},\left\{\left(x_{1}, \ldots, x_{n}\right) \in r: x_{j}>1-\eta / 2\right\}$ whatever the line $r$, parallel to $x_{j}$ axis $(j=1, \ldots, n)$ is.

In this way (see $(4.11),(4.11)^{\prime}$ and $\left.(4.11)^{\prime \prime}\right)$ we have determined, for every arbitrary $\varepsilon>0$, some functions $a_{1}^{\varepsilon}(x), \ldots, a_{n}^{\varepsilon}(x)$ such that:

$$
\begin{gathered}
\left\|a_{i}-a_{i}^{\varepsilon}\right\|_{W^{1,4+2 \alpha}(\Omega)}<\varepsilon ; \\
a_{i}^{\varepsilon} \in C^{\infty}(\bar{\Omega}) ; \quad a_{i}^{\varepsilon}(x) \geqq 1, \quad \forall x ; \\
\forall\left(x_{1}, \ldots, x_{n}\right) \in \bar{\Omega}, \quad \forall j \in\{1, \ldots, n\}, \\
\left(x_{j} \in[0, \eta / 2] \cup[1-\eta / 2,1] \Rightarrow D_{x_{j}} a_{i}^{\varepsilon}\left(x_{1}, \ldots, x_{n}\right)=0\right) .
\end{gathered}
$$

If $\varepsilon$ is ,,sufficiently small" ( $\varepsilon<\varepsilon_{0}$ appropriately chosen), from (4.12) it follows that:

$$
A^{\varepsilon} \equiv \sum_{i, k=1}^{n}\left|D_{x_{k}} a_{i}^{\varepsilon}\right|_{\Omega, 4+2 \alpha}<2 A \equiv 2 \sum_{i, k=1}^{n}\left|D_{x_{k}} a_{i}\right|_{\Omega, 4+2 \alpha},
$$

unless $a_{i}$ is constant $\forall i$ (in which case we apply Proposition (4.I)).

Once (4.10), (4.12) and (4.12)' are achieved, set $\varepsilon=1 / k(k=1,2, \ldots)$, we consider the Dirichlet problem:

$$
\begin{gathered}
\mathscr{L}_{1}^{k} u \equiv-\sum_{i=1}^{n} a_{i}^{1 / k}(x) D_{x_{i}}^{2} u(x)=f_{1 / k}(x) \\
{\left[f_{1 / k} \in C_{0}^{\infty}(\Omega) \subset C_{0}^{\text {even }}(\Omega) ; \sup _{\Omega}\left|f_{1 / k}\right| \leqq \sup _{\Omega}|f|\right]} \\
\left.u\right|_{\partial \Omega}=0 .
\end{gathered}
$$

Since the coefficients $a_{i}^{1 / k}$ verify (4.6) [because of (4.12)], and $f_{1 / k} \in C_{0}^{\infty}(\Omega) \subset$ $\subset C_{0}^{\text {even }}(\Omega) \subset \mathscr{P}^{2 r, 2}(\Omega), \forall r \in N$ [because of $(4.10)$ ] (and, in particular, for $2 r>1+$ $+n / 2)$ by Theorem 4.I, we have:

$$
\begin{gathered}
\exists \mid u_{k} \in \mathscr{P}^{2 r+2,2}(\Omega) \subset C^{3}(\bar{\Omega}): \\
\mathscr{L}_{1}^{k} u_{k} \equiv-\sum_{i=1}^{n} a_{i}^{1 / k} D_{x_{i}}^{2} u_{k}=f_{1 / k} \text { in } \Omega \\
\left.u_{k}\right|_{\partial \Omega}=0 .
\end{gathered}
$$

But the coefficients $a_{i}^{1 / k}$ satisfy (ad abundantiam) the hypothesis of Theorem 3.I and 3.II.

Moreover, if $\varepsilon=1 / k$ is , sufficiently small" $\left(k>1 / \varepsilon_{0}\right)$, the number $\Psi \equiv 2 A$ 
(see $\left.(4.12)^{\prime}\right)$ satisfies the condition:

$$
2 A \equiv \Psi \geqq A^{1 / k} \equiv \sum_{i, j=1}^{n}\left|D_{x_{j}} a_{i}^{1 / k}\right|_{\Omega, 4+2 \alpha} ;
$$

finally, the number $\Phi \equiv \sup _{\Omega}|f|($ see (4.10)) satisfies the condition:

$$
\sup _{\Omega}|f| \equiv \Phi \geqq \sup _{\Omega}\left|f_{1 / k}\right| \equiv \sup _{\Omega}\left|\mathscr{L}_{1}^{k} u_{k}\right|
$$

Thus, writing (3.16) of theorem 3.II (this is allowed because $u_{k} \in \mathscr{U}$, by (4.14)), we have:

$$
\begin{gathered}
\left|D^{2} u_{k}\right|_{\Omega, 2+\alpha}+\left|D^{1} u_{k}\right|_{\Omega, 4+2 \alpha}+\sup _{\Omega}\left|u_{k}\right| \leqq \\
\leqq c_{1}(n, \alpha)\left[\sup _{\Omega}|f|+\left\{c(n) H\left(2 A, \sup _{\Omega}|f|\right)\right\}^{1 /(2+\alpha)}\right], \quad \forall k \in N_{0} .
\end{gathered}
$$

Since, in general, the coefficients $a_{i}$ are not bounded, there are further remarks to make before reaching the assertion. The right-hand side of (4.15) is a constant which does not depend on $k$. Then we can consider a subsequence of $\left\{u_{k}\right\}$ weakly convergent, in $W^{2,2+\alpha}(\Omega)$, towards a function $u \in W^{2,2+\alpha}(\Omega)$ which has zero trace on $\partial \Omega$ (like the $u_{k}$ 's). Without loss of generality, we can assume that this subsequence, still denoted by $\left\{u_{k}\right\}$, strongly converges, in $W^{1,2+\alpha}(\Omega)$, and even almost everywhere in $\Omega$, towards $u$.

Writing for these $u_{k}$ 's (belonging to $\mathscr{U}$ ) formula (3.2) and taking the limit for $k \rightarrow+\infty$, we get:

Moreover, set:

$$
\begin{gathered}
\left.\forall_{k}, \quad \sup _{\Omega}\left|u_{k}\right| \leqq(\mathrm{e}-1) \sup _{\Omega}\left|f_{1 / k}\right| \leqq(\mathrm{e}-1) \sup _{\Omega} \mid f\right) \\
\Rightarrow \sup _{\Omega}|u| \leqq(\mathrm{e}-1) \sup _{\Omega}|f| .
\end{gathered}
$$

$$
\begin{gathered}
q=\frac{n(4+2 \alpha)}{n-4+2|\alpha|}, \quad p=\frac{n(4+2 \alpha)}{n(1+2 \alpha)+(4+2 \alpha)}, \\
p^{\prime}=\frac{p}{p-1} \equiv \frac{n(4+2 \alpha)}{3 n-4+2|\alpha|},
\end{gathered}
$$

by (4.12) we have: $a_{i}^{1 / k} \rightarrow_{k \rightarrow \infty} a_{i} \in L^{q}(\Omega)$, in $L^{q}(\Omega)$ and hence: $\forall \varphi \in L^{p}, \varphi a_{i} \in$ $\in L^{(2+\alpha) /(1+\alpha)} \equiv\left(L^{2+\alpha}\right)^{\prime}$. Besides: $D_{x_{i}}^{2} u_{k} \rightarrow_{k \rightarrow \infty} D_{x_{i}}^{2} u$ weakly in $L^{2+\alpha}$; so we obtain:

$$
\lim _{k \rightarrow \infty} \int_{\Omega} \varphi a_{i}\left(D_{x_{i}}^{2} u_{k}-D_{x_{i}}^{2} u\right) \mathrm{d} x=0, \quad \forall \varphi \in L^{p} .
$$

On the other side, being: $1 / p+1 /(2+\alpha)+1 / q=1$ and remembering (4.15) and the covergence of $\left\{a_{i}^{1 / k}\right\}$ to $a_{i}$ in $L^{q}(\Omega)$, we also have:

$$
\begin{gathered}
\left|\int_{\Omega} \varphi\left(a_{i}^{1 / k}-a_{i}\right) D_{x_{i}}^{2} u_{k} \mathrm{~d} x\right| \leqq \\
\leqq\left\|D_{x_{i}}^{2} u_{k}\right\|_{L^{2+\alpha}}\left\|a_{i}^{1 / k}-a_{i}\right\|_{L^{q}}\|\varphi\|_{L^{p} \rightarrow_{k \rightarrow \infty}} 0, \quad \forall \varphi \in L^{p} .
\end{gathered}
$$


From (4.16) and $(4.16)^{\prime}$ we deduce:

$$
\begin{gathered}
\forall \varphi \in L^{p}(\Omega), \\
\lim _{k \rightarrow \infty} \int_{\Omega}\left(a_{i}^{1 / k} D_{x_{i}}^{2} u_{k}\right) \varphi \mathrm{d} x=\int_{\Omega}\left(a_{i} D_{x_{i}}^{2} u\right) \varphi \mathrm{d} x .
\end{gathered}
$$

After taking the weak limit in $L^{p^{\prime}}(\Omega)$ in both sides of the equation:

$$
\mathscr{L}_{1}^{k} u_{k} \equiv-\sum_{i=1}^{n} a_{i}^{1 / k} D_{x_{i}}^{2} u_{k}=f_{1 / k} \quad(\sec (4.14))
$$

we get the assertion since $f_{1 / k} \rightarrow_{k} f$ in $L^{2}(\Omega)$ (see (4.10)) and $p^{\prime}<2$ (because $n \geqq 4$ ).

q.e.d.

\section{SOME PRELIMINARY}

In order to study some particular equations we shall recall some definitions.

Definition 5.I. (see [6], [13]). Let $u(x)$ be a measurable real function in the bounded open set $G$ of $R^{n}$. We define as distribution function of $u$ and spherically symmetric rearrangement of $u$, respectively, the following functions:

$$
\begin{gathered}
\mu(t)=\operatorname{meas}\{x \in G:|u(x)|>t\} \equiv \text { meas } G_{u}^{t}, \quad t \geqq 0 \\
u^{*}(x)=\inf \left\{t \geqq 0: \mu(t)<C_{n}|x|^{n}\right\}, x \in G^{*},
\end{gathered}
$$

where: $C_{n}=\pi^{n / 2} / \Gamma(1+n / 2)$ is the measure of the unitary ball in $R^{n}$ and $G^{*}$ is the ball in $R^{n}$ which has the origin as center and the same measure as $G$.

The Lorenz Spaces $L(p, q, G)$ and $L(p, \infty, G)$ are defined by:

$$
\begin{gathered}
u \in L(p, q, G) \Leftrightarrow\|u\|_{p, q, G} \equiv \\
\equiv\left[\int_{G^{*}}\left[u^{*}(x)|x|^{n / p}\right]^{q}|x|^{-n} \mathrm{~d} x\right]^{1 / q}<+\infty \\
(1<p<+\infty, 1 \leqq q<+\infty) \\
u \in L(p, \infty, G) \Leftrightarrow\|u\|_{p, \infty, G} \equiv \sup _{G^{*}}\left[|x|^{n / p} u^{*}(x)\right]<+\infty \\
(1<p<+\infty) .
\end{gathered}
$$

It is well known that the following properties hold (see [13]):

$$
\begin{gathered}
\int_{G} u(x) v(x) \mathrm{d} x \leqq \int_{G^{*}} u^{*}(x) v^{*}(x) \mathrm{d} x, \\
\int_{G}|u(x)|^{p} \mathrm{~d} x=\int_{G^{*}}\left|u^{*}(x)\right|^{*} \mathrm{~d} x, \quad \forall p>0
\end{gathered}
$$

where $u, v$ are measurable real functions in $G$.

Let $\Omega$ be the unitary ball in $R^{n}$ with center in the origin [so that $\Omega^{*}=\Omega$ ] and $b(x)$ the function:

$$
\begin{gathered}
b(x) \equiv b_{h}(x)=-2 \beta\left(\cos |x|^{-2 \beta}\right) x_{h}|x|^{-2-2 \beta} \quad x \in \Omega-\{0\}, \\
b(0)=0,
\end{gathered}
$$

where: $0<\beta<1,|x|^{2}=x_{1}^{2}+x_{2}^{2}+\ldots+x_{n}^{2}, h=1, \ldots, n$. For $\left.\theta \in\right] 0, \pi / 2[$, we 
consider the circular cones:

$$
\begin{gathered}
\Lambda_{\theta}=\left\{x \in \mathbb{R}^{n}: x_{h}>|x| \cos \theta>0\right\}, \\
\Omega_{\theta}=\Omega \cap \Lambda_{\theta} .
\end{gathered}
$$

Evidently we have:

$$
\begin{gathered}
\forall t \geqq 0, \quad\{x \in \Omega:|b(x)|>t\} \supseteq \\
\supseteq\left\{x \in \Omega_{\theta}:\left|\cos \frac{1}{|x|^{2 \beta}}\right| \frac{x_{h}}{|x|^{2+2 \beta}}>\frac{t}{2 \beta}\right\} \supseteq \\
\supseteq\left\{x \in \Omega_{\theta}:\left(\cos \frac{1}{|x|^{2 \beta}}\right) \frac{\cos \theta}{|x|^{1+2 \beta}}>\frac{t}{2 \beta}\right\} \supseteq \\
\supseteq\left\{x \in \Omega_{\theta}: \cos \frac{1}{|x|^{2 \beta}}>\frac{1}{2}\right\} \cap\left\{x \in \Omega_{\theta}: \frac{1}{|x|^{1+2 \beta}}>\frac{t}{\beta \cos \theta}\right\} .
\end{gathered}
$$

Hence:

$$
\begin{gathered}
\forall t \geqq 0, \quad\{x \in \Omega:|b(x)|>t\} \supseteq \\
\left.\supseteq\left[\bigcup_{k \in N} x \in \Omega_{\theta}: 2 k \pi-\frac{\pi}{3}<\frac{1}{|x|^{2 \beta}}<2 k \pi+\frac{\pi}{3}\right\}\right] \cap \\
\cap\left\{x \in \Omega_{\theta}: \frac{1}{|x|}>\gamma_{\beta \theta} t^{1 /(1+2 \beta)}\right\},
\end{gathered}
$$

where: $N=\{0,1,2, \ldots\} ; \gamma_{\beta \theta}=(\beta \cos \theta)^{-1 /(1+2 \beta)}$. We shall use the following:

Lemma 5.I. There exist three positive numbers: $r \in] 0,1\left[, A_{\beta, \theta}, \varepsilon=2 \beta(\eta-1)\right.$ : $:(n+2 \beta)$, such that, in the ball $S_{0, r}(n \geqq 2)$, with center in the origin and radius $r$, the function $b(x)$ of (5.3) satisfies the inequality:

$$
\forall x \in S_{0, r}-\{0\}, \quad b^{*}(x) \geqq \frac{A_{\beta, \theta}}{|x|^{1+\varepsilon}} .
$$

Proof. Denoting by $\omega_{\theta}$ the measure of $\Omega_{\theta}$, from (5.5) it follows that:

$$
\begin{aligned}
& \geqq \text { meas } \underset{(2 k \pi-\pi / 3)^{1 / 2 \beta>\gamma_{\beta \theta} t^{1 /(1+2 \beta)}}}{k \in N_{0}}\left\{x \in \Lambda_{0}:\left(2 k \pi-\frac{\pi}{3}\right)^{-1 / 2 \beta}>|x|>\left(2 k \pi+\frac{\pi}{3}\right)^{-1 / 2 \beta}\right\}= \\
& \left.=\omega_{\theta} \sum_{(2 k \pi-\pi / 3)^{1 / 2 \beta>\gamma_{\beta \theta} t^{1 /(1+2 \beta)}}}^{k \in N_{0}}\left[\left(2 k \pi-\frac{\pi}{3}\right)^{-n / 2 \beta}-\left(2 k \pi+\frac{\pi}{3}\right)^{-n / 2 \beta}\right]^{1}\right)
\end{aligned}
$$

because the points $x \in \Lambda_{\theta}$. which belong to the set in the right-hand side trivially satisfy:

$$
\frac{1}{|x|}>\left(2 k \pi-\frac{\pi}{3}\right)^{1 / 2 \beta}>\gamma_{\beta \theta} t^{1 /(1+2 \beta)},
$$

1) We explicitely remark that the union is taken over mutually disjoint sets. 
as well as: $x \in \Omega_{\theta}$ (since $\left.k \in N_{0}=\{1,2, \ldots\}\right)$. From (5.5)' it follows:

$(5.5)^{\prime \prime} \forall t \geqq 0, \mu(t) \geqq \frac{n \pi \omega_{\theta}}{3 \beta} \sum_{k>(1 / 6)+\left(\gamma_{\left.\beta \theta^{2 \beta} / 2 \pi\right) t^{2 \beta /(1+2 \beta)}}\left(2 k \pi+\frac{\pi}{3}\right)^{-1-(n / 2 \beta)}\right.} \geqslant$
$\geqq a_{\beta \theta} \sum_{k \geqq 1+c^{\prime} \beta t^{2 \beta /(1+2 \beta)}} k^{-(n+2 \beta) / 2 \beta}$

where:

$$
c_{\beta \theta}^{\prime}=\max \left\{1, \frac{1}{2 \pi} \gamma_{\beta \theta}^{2 \beta}\right\}, \quad a_{\beta \theta}=\frac{n \pi \omega_{\theta}}{3 \beta(4 \pi)^{1+n / 2 \beta}} .
$$

We observe that, since $c_{\beta \theta}^{\prime}>0$, in particular we have:

(5.6) $0 \leqq t \leqq 1 \Rightarrow a_{\beta \theta} \sum_{k \geqq 1+c^{\prime} \beta \theta^{2 \beta /(1+2 \beta)}} k^{-(n+2 \beta) / 2 \beta} \geqq a_{\beta \theta} \sum_{k \geqq 1+c^{\prime} \beta \theta} k^{-(n+2 \beta) / 2 \beta} \geqq \sigma_{\beta \theta}$.

From $(5.5)^{\prime \prime}$ and (5.6) we deduce:

$$
\forall t \geqq 0, \quad\left\{\mu(t)<s \leqq \frac{1}{2} \sigma_{\beta \theta} \Rightarrow t>1\right\} .
$$

On the other hand, being $c_{\beta \theta}^{\prime} \geqq 1,(5.5)^{\prime \prime}$ also implies:

(5.8) $\forall t>1, \mu(t)>a_{\beta \theta} \sum_{2 c^{\prime} \beta \theta t^{2 \beta /(1+2 \beta)} \leqq k \leqq 4 c^{\prime} \beta t^{2 \beta /(1+2 \beta)}} k^{-(n+2 \beta) / 2 \beta}>a_{\beta \theta}^{\prime} t^{-(n+2 \beta) /(1+2 \beta)}$,

where:

Moreover:

$$
a_{\beta \theta}^{\prime}=a_{\beta \theta}\left(4 c_{\beta \theta}^{\prime}\right)^{-(n+2 \beta) / 2 \beta} \text {. }
$$

$$
|x| \leqq r \equiv \min \left\{1,\left[\frac{\sigma_{\beta \theta}}{2 c_{n}}\right]^{1 / n}\right\} \Rightarrow c_{n}|x|^{n} \leqq \frac{1}{2} \sigma_{\beta \theta} .
$$

Thus, in virtue of (5.7) and (5.8) we have:

and hence:

$$
\begin{aligned}
& \forall x \in S_{0, r}-\{0\}, \quad\left\{t \geqq 0: \mu(t)<c_{n}|x|^{n}\right\} \subset \\
& \subset\left\{t>1: a_{\beta \theta}^{\prime} t^{-(n+2 \beta) /(1+2 \beta)}<c_{n}|x|^{n}\right\} \subset \\
& \subset\left\{t>0: a_{\beta \theta}^{\prime} t^{-(n+2 \beta) /(1+2 \beta)}<c_{n}|x|^{n}\right\},
\end{aligned}
$$

$$
\begin{gathered}
\forall x \in S_{0, r}-\{0\}, \\
b^{*}(x) \equiv \inf \left\{t \geqq 0: \mu(t)<c_{n}|x|^{n}\right\} \geqq
\end{gathered}
$$

$\geqq \inf \left\{t>0: a_{\beta \theta}^{\prime} t^{-(n+2 \beta) /(1+2 \beta)}<c_{n}|x|^{n}\right\}=\left(a_{\beta \theta}^{\prime} / c_{n}\right)^{(1+2 \beta) /(n+2 \beta)}:|x|^{(n+2 n \beta) /(n+2 \beta)}$.

From (5.10) the assertion follows taking:

$$
\begin{gathered}
A_{\beta \theta}=\left(a_{\beta \theta}^{\prime} / c_{n}\right)^{(1+2 \beta) /(n+2 \beta)}, \\
r=\min \left\{1,\left[\frac{\sigma_{\beta \theta}}{2 c_{n}}\right]^{1 / n}\right\}, \varepsilon=\frac{2 \beta(n-1)}{n+2 \beta} .
\end{gathered}
$$

Now we consider the Dirichlet problem:

$$
\begin{gathered}
L_{0} u \equiv \sum_{i, j=1}^{n} a_{i j}(x) D_{x_{j} D_{x_{j}}} u(x)=f(x) \text { in } G \\
\left.u\right|_{\partial G} \approx 0,
\end{gathered}
$$


where:

$$
m_{0}|\xi|^{2} \leqq \sum_{i, j} a_{i j}(x) \xi_{i} \xi_{j} \leqq m_{1}|\xi|^{2}, \quad \forall x \forall \xi ; \quad m_{0}>0 .
$$

Recently, for the problem (5.11) an existence and uniqueness theorem has been established by A. Alvino and G. Trombetti in [2], under the hypothesis:

$$
\begin{gathered}
D_{x_{h}} a_{i j} \in L(n, \infty, G) \\
{\left[\Leftrightarrow\left\|D_{x_{h}} a_{i j}\right\|_{n, \infty, G} \equiv \sup _{G^{*}}\left[|x|\left(D_{x_{h}} a_{i j}\right)^{*}(x)\right]<+\infty\right] .}
\end{gathered}
$$

Also P. Buonocore has obtained a similar theorem under the assumption (see [3]):

$$
\begin{gathered}
D_{x_{h}} a_{i j} \in L(n, q, G) ; n<q \\
{\left[\Leftrightarrow\left(\int_{G^{*}}\left[\left(D_{x_{h}} a_{i j}\right)^{*}(x)|x|\right]^{q}|x|^{-n} \mathrm{~d} x\right)^{1 / q}<+\infty ; n<q\right] .}
\end{gathered}
$$

In both theorems the authors suppose $\partial G \in C^{3}$. We also recall the well known existence and uniqueness Theorem for (5.11) [in $\left.W_{\gamma_{0}}^{2,2}(G) \equiv\left\{u \in W^{2,2}:\left.u\right|_{\partial G}=0\right\}\right]$ of $G$. Talenti (see [14], Theorem 1.1) obtained under the assumptions:

a) $\partial G$ belongs to $C^{3}$; considered the outher normal to $\partial G$, the mean curvature of $\partial G$ has non positive constant sign;

b) $\sum_{k=1}^{n} a_{k k}(x)=1, \sum_{i, k=1}^{n} a_{i k}^{2}(x) \leqq 1 /(n-1+\varepsilon), 0<\varepsilon<1$

$\left[\Leftrightarrow \dot{n}-\left(\sum_{i} a_{i i}\right)^{2}\left(\sum_{i j} a_{i j}^{2}\right)^{-1} \leqq k^{2}<1\right.$ (Cordes) $] ;$

c) $f \in L^{2}(G)$.

Finally, we recall the basic existence Theorem of C. Miranda (Theor. 5.V of [8]) [Theorem 4.II of this paper is an extension of it] obtained for the equation:

$$
\begin{gathered}
L u \equiv \sum_{i, j=1}^{n} a_{i j}(x) D_{x_{i}} D_{x_{j}} u+\sum_{i=1}^{n} b_{i}(x) D_{x_{i}} u+c(x) u=f, \\
f \in L^{\infty}(\Omega), \quad u \in W_{\gamma_{0}}^{2,2}(\Omega) \cap L^{\infty}(\Omega),
\end{gathered}
$$

under the hypotheses:

$$
\begin{gathered}
a_{i j} \in W^{1, \lambda}(\Omega), \quad b_{i} \in L^{\lambda}(\Omega), \quad c \in L^{\lambda / 2}(\Omega) \text { with } 4 \leqq \lambda<n ; \\
m_{0}|\xi|^{2} \leqq \sum_{i j} a_{i j}(x) \xi_{i} \xi_{j} \leqq m_{1}|\xi|^{2}, \quad \forall x \forall \xi \quad\left(m_{0}>0\right), \quad c(x) \leqq c_{0}<0 ; \\
\partial G \text { belongs to } C^{3} .
\end{gathered}
$$

Moreover, if $n=4$, an existence and uniqueness Theorem is established in [8] 1.V under the assumptions (5.15) written for $\lambda=n=4$.

In this case the function $f$ of $(5.14)$ can be taken in $L^{q}(q \geqq 2)$ and the unique solution of the equation $L u=f$ belongs to $W_{\gamma_{0}}^{2,2}(\Omega)$.

A ,sharp" procedure to test a theory is the following: we ,force", $)$ it to ,,decode" the information hidden in a certain equation of ,,reserved character".

This is what we will try to do in the next section.

2) "to force" = "astringere" (in italian). 


\section{A TEST EQUATION}

We study the Dirichlet problem for a particular elliptic equation whose coefficients are not continuous:

$$
\begin{gathered}
-\sum_{i=1}^{n} a_{i}(x) D_{x_{i}}^{2} u+l u \equiv \\
\equiv-\sum_{i=1}^{n} K_{i}\left(1+K_{i}^{-1}+\operatorname{sen}\left[\left|x-x^{i}\right|^{-2 \beta_{i}}\right]\right) D_{x_{i}}^{2} u+l u=f \text { in } \Omega, \\
\left.u\right|_{\partial \Omega}=0,
\end{gathered}
$$

where: $n \geqq 4 ; \Omega$ is a bounded open set in $R^{n} ; k_{i}$ and $\beta_{i}$ are positive constants; $l$ is a non negative constant and $x^{i}=\left(x_{1}^{i}, \ldots, x_{n}^{i}\right) \in \Omega$.

Our aim is to analyse the information about (6.1) that we can deduce from the theories mentioned at the end of the previous section varying the parameters $k_{i}$ and $\beta_{i}$.

Proposition 6.I. (i) If $\partial \Omega \in C^{3}, l=0$, considered the outer normal to $\partial \Omega$, the mean curvature of $\partial \Omega$ has non positive constant sign and the coefficients $a_{i}$ of (6.1) satisfy Cordes's condition [for example, if $\sum_{i=1}^{n}\left(1+2 K_{i}\right)^{2}<\left(n^{2}-n\right) /\left(n-1-k^{2}\right)$ with $k^{2}<1$, whatever $\beta_{i}$ are], then according to theorem 1.1 of [14], problem (6.1) has a unique solution $u \in W^{2,2}(\Omega)$, for any fixed $f \in L^{2}(\Omega)$.

(ii) If Cordes's condition does not hold [that is if the $k_{i}$ 's are arbitrary positive numbers], if $\partial \Omega \in C^{3}$ and:

$$
l>0 ; n>4 ; 0<\beta_{i}<(n-4) / 8,
$$

then, by theorem $5 . \mathrm{V}$ of [8], problem (6.1) has at least one solution $u \in W^{2,2}(\Omega) \cap$ $\cap L^{\infty}(\Omega)$, for any fixed $f \in L^{\infty}(\Omega)$.

If , instead, $n=4$ and $\beta_{i}>0$ nothing can be said according to theorem $1 . \mathrm{V}$ of $[8]$.

(iii) If the $k_{i}$ 's are arbitrary, $\left.\Omega=\right] 0,1\left[{ }^{n}\right.$ and:

$$
l=0 ; \quad n \geqq 4 ; \quad 0<\beta_{i}<\frac{n-4+2|\alpha|}{8+4 \alpha}
$$

with: $-1 / 6 \leqq \alpha<0$, then, by theorem 4.II of this paper, problem (6.1) has at least one solution $u \in W^{2,2+\alpha}(\Omega) \cap L^{\infty}(\Omega)$, for any fixed $f \in L^{\infty}(\Omega)$.

Finally, if $\Omega=\left\{x \in \mathbb{R}^{n}:|x|<1\right\}, x^{i}=0 \forall i, \beta_{i}>0$, nothing can be said about problem (6.1) according to the results of [2] and [3] (unless $k_{1}=\ldots=k_{n}$ and $\left.\beta_{1}=\ldots=\beta_{n}\right)$.

Proof. It is obvious that in the case (i) the conditions (5.13) hold, so that Theorem 1.1 of [14] applies.

We also observe that, for the equation (6.1) we have:

$$
\forall x \forall i, \quad 1 \leqq a_{i}(x) \leqq 1+2 k_{i},
$$


so that:

$$
\begin{gathered}
\sum_{i=1}^{n}\left(1+2 k_{i}\right)^{2}<\frac{n^{2}-n}{n-1-K^{2}} \quad\left(\text { with } K^{2}<1\right) \Rightarrow \\
\Rightarrow \frac{\left(\sum_{i} a_{i}\right)^{2}}{\sum_{i} a_{i}^{2}} \equiv 1+\frac{\sum_{i \neq j \leqq n} a_{i} a_{j}}{\sum_{i} a_{i}^{2}} \geqq 1+\frac{n^{2}-n}{\sum_{i}\left(1+2 k_{i}\right)^{2}}>n-K^{2},
\end{gathered}
$$

which is precisely Cordes's condition.

In (ii) case, to apply Theorem $5 . \mathrm{V}$ (or theorem $1 . \mathrm{V}$ if $n=4$ ) of [8] [see (5.15)] it is sufficient to have:

$$
D_{x_{h}} a_{i} \equiv-2 k_{i} \beta_{i}\left(\cos \left[\left|x-x^{i}\right|^{-2 \beta_{i}}\right]\right) \frac{x_{h}-x_{h}^{i}}{\left|x-x^{i}\right|^{2+2 \beta_{i}}} \in L^{4}(\Omega) .
$$

But, if $n>4,(6.2)$ implies certainly $(6.2)^{\prime}[\Rightarrow(5.15)]$ and problem (6.1) has at least one solution $u \in W^{2,2}(\Omega) \cap L^{\infty}(\Omega)$ for any fixed $f \in L^{\infty}(\Omega)$. If, instead, $n=4, \beta_{i}>0$, and, for sake of simplicity, $\Omega=\left\{x \in \mathbb{R}^{n}:|x|<1\right\} \equiv \Omega^{*}$ and $x^{i}=0 \forall_{i}$, we will show that the first of the conditions (5.15), written with $\lambda=4$, cannot hold, so that Theorem 1.V of [8] cannot be applied.

Actually, for $x^{i}=0$ and $\beta_{i}>0, D_{x_{h}} a_{i}(x)$ coincides (up to the factor $k_{i}$ ) with the function $b(x) \equiv b_{h}(x)$ of (5.3) [with $\beta=\beta_{i}$ ]. Consequently, by (5.3)* of Lemma 5.I and the second of (5.2), we have:

$$
\begin{gathered}
\int_{\Omega}\left|D_{x_{h}} a_{i}(x)\right|^{4} \mathrm{~d} x=\int_{\Omega^{*}}\left|\left(D_{x_{h}} a_{i}\right)^{*}(x)\right|^{4} \mathrm{~d} x \geqq \\
\geqq\left(k_{i} A_{\beta_{i} \theta}\right)^{4} \int_{S_{0, r}}|x|^{-4-4 \varepsilon} \mathrm{d} x=+\infty, \quad\left(\varepsilon=\frac{6 \beta_{i}}{4+2 \beta_{i}}\right),
\end{gathered}
$$

so that: $a_{i} \notin W^{1,4}(\Omega)$ and Theorem 1 .V of [8] cannot be applied.

Let us consider the (iii) case. In order to apply Theorem 4.II, we should have:

$$
\left.D_{x_{h}} a_{i} \in L^{4+2 \alpha}(\Omega), \quad \text { with: }-1 / 6 \leqq \alpha<0, \quad \Omega=\right] 0,1\left[{ }^{n}, \quad n \geqq 4 .\right.
$$

But (6.3) implies, evidently, (6.3)'. Thus, by Theorem 4.II, problem (6.1) has at least one solution $u \in W^{2,2+\alpha}(\Omega) \cap L^{\infty}(\Omega)$, for any fixed $f \in L^{\infty}(\Omega)$.

In the (iv) case we have: $D_{x_{h}} a_{i}(x)=k_{i} b_{h}(x)$ [that is the function (5.3) with $\beta=\beta_{i}$ ]. Therefore by $(5.3)^{*}$ of Lemma 5.I we get:

$$
\begin{gathered}
\forall n, \forall q>n, \quad\left(\int_{\Omega^{*}}\left[\left(D_{x_{h}} a_{i}\right)^{*}(x)|x|\right]^{q}|x|^{-n} \mathrm{~d} x\right)^{1 / q} \geqq \\
\geqq\left(\int_{S_{0, r}}\left(k_{i} A_{\beta_{i} \theta}\right)^{q}|x|^{-q-q \varepsilon}|x|^{q-n} \mathrm{~d} x\right)^{1 / q}=+\infty, \quad\left(\varepsilon=\frac{2 \beta_{i}(n-1)}{n+2 \beta_{i}}\right) .
\end{gathered}
$$

Consequently, since (5.12)' does not hold, the results of [3] cannot be applied. In the same way, since (5.12) does not hold, the results of [2] cannot be applied. In fact, by $(5.3)^{*}$ we have:

$$
|x|\left(D_{x_{h}} a_{i}\right)^{*}(x) \equiv k_{i}|x| b_{h}^{*}(x) \geqq k_{i} A_{\beta_{i} \theta}|x|^{-\varepsilon}, \quad \text { in } S_{0, r} \text { q.e.d. }
$$


Remark 6.I. In [12] Christian G. Simader develops a $L^{p}$ theory for the (variational) Dirichlet problem based on a generalization of Gårding's inequality.

Let $G$ be a bounded open set in $R^{n}$ with boundary $\partial G \in C^{m}(m \geqq 1)$, and:

$$
\begin{gathered}
B[\varphi, \psi] \equiv \sum_{\substack{|\alpha| \leqq m \\
|\beta| \leqq m}} \int_{G} a_{\alpha \beta} D^{\alpha} \varphi D^{\beta} \psi \mathrm{d} x \\
\left(a_{\alpha \beta} \in L^{\infty}(G) ; a_{\alpha \beta} \in C^{0}(\bar{G}) \text { if }|\alpha|=|\beta|=m\right)
\end{gathered}
$$

a regular uniformly elliptic bilinear form of Dirichlet type. Then $B[\varphi, \psi]$ is continuous and coercive:

$$
\begin{aligned}
& |B[u, v]| \leqq c\|u\|_{W^{m, p}(G)}\|v\|_{W^{m, q}(G)} \\
& \forall(u, v) \in \stackrel{\circ}{W}^{m, p}(G) \times{\stackrel{\circ}{W^{m}, q}}^{(G)}, \frac{1}{p}+\frac{1}{q}=1, \\
& \sup _{\substack{\varphi \in W^{m, q}(G) \\
\|\varphi\|_{W^{m, q}}^{\circ}=1}}|B[u, \varphi]| \geqq c_{1}\|u\|_{W^{m, p}(G)}-c_{2}\|u\|_{L^{p}(G)} \\
& \forall u \in \stackrel{\circ}{W}^{m, p}(G), \quad \frac{1}{p}+\frac{1}{q}=1
\end{aligned}
$$

(see Definition (5.1), Definition 6.2 and Theorem 6.3 of [12]). Let $\mathscr{L}$ be an elliptic operator (of order $2 m$ ) and $B[u, v]$ a bilinear form "equivalent" to $\mathscr{L}$ in the following meaning:

$$
\int_{G} \mathscr{L} \varphi \psi \mathrm{d} x=B[\varphi, \psi], \quad \forall \varphi, \psi \in C_{0}^{\infty}(G) .
$$

The homogeneous "weak" Dirichlet problem is the following:

$$
B[u, \varphi]=\int_{G} f \varphi \mathrm{d} x \quad \forall \varphi \in C_{0}^{\infty}(G), \text { with } f \in L^{p}(G), \quad u \in \stackrel{\circ}{W}^{m, p}(G) .
$$

For such a problem, if $B$ is regular and uniformly elliptic, some regularization theorems (Theorem 9.11 and 11.2 of [12]) and an existence theorem (Theorem 10.7) are established.

We wonder if it would be possible a variational approach (in the sense above precised) for an operator $\mathscr{L} \equiv-\sum_{i} a_{i}(x) D_{x_{i}}^{2}$ satisfying (4.1) in a bounded open set $\Omega$ with $\partial \Omega \in C^{1}$. First of all we need a bilinear form equivalent to $\mathscr{L}$ (see (6.6)). This can be done, in the most natural way, multiplying $\mathscr{L} u$ by $v\left(u, v \in C_{0}^{\infty}(\Omega)\right)$ and integrating by parts:

$$
\begin{gathered}
\int_{\Omega} \mathscr{L} u . v \mathrm{~d} x=B_{\mathscr{L}}[u, v] \equiv \sum_{i=1}^{n} \int_{\Omega} a_{i} D_{x_{i}} u D_{x_{i}} v \mathrm{~d} x+ \\
+\sum_{i=1}^{n} \int_{\Omega} D_{x_{i}} a_{i} D_{x_{i}} u . v \mathrm{~d} x, \quad \forall u, v \in C_{0}^{\infty}(\Omega) .
\end{gathered}
$$

But here $B_{\mathscr{L}}$ is not a regular form in the sense of Definition 6.2 of [12], because, in general, being, for $(4.1)$ :

$$
a_{i} \in W^{1,4+2 \alpha}(\Omega), \quad-\frac{1}{6} \leqq \alpha<0, \quad \Omega \subset \mathbb{R}^{n}, \quad n \geqq 4,
$$


we only have:

$$
D_{x_{i}} a_{i} \in L^{4+2 \alpha}(\Omega), \quad a_{i} \in L^{n(4+2 \alpha) /(n-4+2|\alpha|)}(\Omega),
$$

and not:

$$
D_{x_{i}} a_{i} \in L^{\infty}(\Omega), \quad a_{i} \in C^{0}(\bar{\Omega})
$$

(as required in Definition 6.2).

Therefore for the problem (6.7) relative to $B_{\mathscr{L}}$ it is not possible to use the Theorem 10.7 of [12].

More than that, this is true for any bilinear form equivalent to $\mathscr{L}$ (in the sense of (6.6)), since it would be easy to show that for such a form it should necessarily be: $a_{\beta \beta} \in\left\{a_{1}, \ldots, a_{n}\right\}$ for $|\beta|=1$, while the coefficients $a_{i}$ of $\mathscr{L}$ belong to $W^{1,4+2 \alpha}(\Omega)$ [and not to $C^{0}(\bar{\Omega})$ or $\left.L^{\infty}(\Omega)\right]$ so that $B[u, v]$ is even meaningless for $(u, v) \in W^{1, p} \times$ $\times W^{1, q}(1 / p+1 / q=1)$.

From all this we deduce that the variational approach (either in $L^{p}$ or in $L^{2}$ ) is not possible for an operator $\mathscr{L}$ satisfying (4.1). Obviously the variational methods (of Simader-Agmon type) can be applied (in place of the continuation method) to the approximating problems:

$$
\mathscr{L}_{1}^{k} u_{k}=f_{1 / k},\left.\quad u_{k}\right|_{\partial \Omega}=0,
$$

considered in the proof of theorem 4.II, where $f_{1 / k}$ converges to $f$ and the coefficients of $\mathscr{L}_{1}^{k}$ to those of $\mathscr{L}\left[\right.$ in $\left.W^{1,4+2 \alpha}\right]$.

This is possible (except for the problems concerning $\partial \Omega$, if $\Omega=] 0,1\left[{ }^{n}\right.$ ) because the coefficients of $\mathscr{L}_{1}^{k}$ are regular, unlike those of $\mathscr{L}$. But after this, to reach a solution of the limit problem: $\mathscr{L} u=f,\left.u\right|_{\partial \Omega}=0$, we cannot use the estimates for the $u_{k}$ 's furnished by Simader's theory, where the constants depend on the moduli of continuity of the coefficients (see Lemma 9.2 page 138 of [12] as well as Lemma 9.11 and Theorem 10.10).

Instead we need to use the new estimates introduced in the section 3 of this paper, as we do in the proof of theorem 4.II.

Remark 6.II. Equation (6.1) has the coefficients $a_{i}$ bounded. But the boundedness of the coefficients is not necessary to verify the hypotheses of (4.1).

For instance the Existence Theorem 4.II can be applied to the equation:

$$
-\sum_{i=1}^{n}\left(1+\left|x-x^{i}\right|^{-2 \beta_{i}}\left[1+\operatorname{sen}\left(\left|x-x^{i}\right|^{-2 \beta_{i}}\right)\right]\right) D_{x_{i}}^{2} u(x)=f(x), \text { in } \Omega,
$$

where: $\Omega=] 0,1\left[n ; n \geqq 4 ; x^{i}=\left(x_{1}^{i}, \ldots, x_{n}^{i}\right) \in \Omega ; 0<\beta_{i}<(n-4+2|\alpha|) /(16+8 \alpha)\right.$, with: $-1 / 6 \leqq \alpha<0$. In particular, for $n=4$ and $\alpha=-1 / 6$, we can take $2 \beta_{i} \in$ $\in] 0, \frac{1}{22}[$.

Another equation to which to apply Theorem 4.II is the following:

$$
\begin{gathered}
-\sum_{i=1}^{n}\left[1+\sum_{j=1}^{\infty} \frac{1+\operatorname{sen}\left(\left|x-x^{i j}\right|^{-2 \beta_{i j}}\right)}{k_{i j} 2^{j}\left|x-x^{i j}\right|^{2 \beta_{i j}}}\right] D_{x_{i}}^{2} u(x)=f(x), \\
\text { in } \Omega \equiv] 0,1\left[^{n},\right.
\end{gathered}
$$


where: $n \geqq 4 ;\left\{x^{i j}\right\}_{j \in N_{0}} \equiv\left\{\left(x_{1}^{i j}, \ldots, x_{n}^{i j}\right)\right\}_{j \in N_{0}}$ is a dense sequence in $\Omega$;

$$
0<\beta_{i j}<\frac{n-4+2|\alpha|}{16+8 \alpha} \text {, }
$$

with:

$$
-1 / 6 \leqq \alpha<0 ; \quad k_{i j}=\left\|\frac{1+\operatorname{sen}\left(\left|x-x^{i j}\right|^{-2 \beta_{i j}}\right)}{\left|x-x^{i j}\right|^{\beta_{i j}}}\right\|_{W^{1,4+2 \alpha(\Omega)}} .
$$

Each coefficient of the equation (6.10) has infinitely many singular points in any neighborhood contained in $\Omega$.

It is also possible to build examples of equations (to which to apply Theorem 4.II) where, unlike for $(6.1),(6.9)$ and $(6.10)$, the singular points of the coefficients fill in whole manifolds of positive dimension.

If $n \geqq 5$, we consider (for $i=1, \ldots, n$ ) the straight line $r_{i}$ parallel to the $x_{i}$ axis and passing through the point $\left(x_{1}^{i}, \ldots, x_{i-1}^{i}, 0, x_{i+1}^{i}, \ldots, x_{n}^{i}\right) \in \partial \Omega$ and denote by

$$
d_{i}(x)=\left[\sum_{j \neq i}^{1 \leqq j \leqq n}\left(x_{j}-x_{j}^{i}\right)^{2}\right]^{1 / 2}
$$

the distance, from $r_{i}$, of the generic $\left.x \in \Omega \equiv\right] 0,1\left[{ }^{n}\right.$. The theorem 4 .II can be applied to the equation:

$$
-\sum_{i=1}^{n}\left(1+d_{i}(x)^{-2 \beta_{i}}\left[1+\operatorname{sen}\left(d_{i}(x)^{-2 \beta_{i}}\right)\right]\right) D_{x_{i}}^{2} u(x)=f(x), \text { in } \Omega,
$$

if: $0<\beta_{i}<(n-5+2|\alpha|) /(16+8 \alpha),-\frac{1}{6} \leqq \alpha<0$.

\section{References}

[1] R. A. Adams: Sobolev Spaces. Academic Press, 1975.

[2] A. Alvino, G. Trombetti: Second order elliptic equations whose coefficients have their first derivatives weakly- $L^{n}$. Ann. Mat. Pura Appl. (IV), Vol. CXXXVIII, pp. 331-340, 1984.

[3] P. Buonocore: Second order elliptic equations whose coefficients have their first derivatives in the Lorenz spaces. Mathematical Depart. of Naples University, Preprint n. 41, 1983.

[4] E. Gagliardo: Ulteriori proprietà di alcune classi di funzioni in più variabili. Ricerche di Matematica, Vol. VIII, pp. 24-51, 1959.

[5] D. Gilbarg, N. S. Trudinger: Elliptic partial differential equations of second order. SpringerVerlag, Berlin, 1977.

[6] G. H. Hardy, J. E. Littlewood, G. Polya: Inequalities. Cambridge University Press, 1964.

[7] C. Miranda: Su alcune diseguaglianze integrali. Memorie Accademia dei Lincei, 7, pp. 1-14, 1963.

[8] C. Miranda: Sulle equazioni ellittiche del secondo ordine di tipo non variazionale a coefficienti discontinui. Ann. Mat. Pura Appl. 63, pp. 353-386, 1963.

[9] C. Miranda: Equazioni lineari ellittiche di tipo non variazionale. Rend. Sem. Mat.Milano, 32, 1963.

[10] J. Nečas: Les méthodes directes en théorie des équationes elliptiques. Masson et Cie, Editeurs Paris, Academia Editeurs Prague, 1967. 
[11] L. Nirenberg: On elliptic partial differential equations. Annali Sc. Normale Sup. Pisa, 13, pp. 123-131, 1959.

[12] C. G. Simader: On Dirichlet's boundary value problem. Springer-Verlag, Berlin, 1972.

[13] G. Talenti: Elliptic equations and rearrangements. Annali Sc. Normale Sup. Pisa, 3, pp. 697-718, 1976.

[14] G. Talenti: Sopra una classe di equazioni ellittiche a coefficienti misurabili. Ann. Mat. Pura Appl. 69, pp. 285-304, 1965.

[15] M. Troisi: Teoremi di inclusione per spazi di Sobolev non isotropi. Ricerche di Matematica, Vol. XVIII, pp. 3-24, 1969.

Author's address: Via Francesco Netti 67, 80131 Napoli, Italy. 